Revue des patrimoines

\title{
La reconstruction du village témoin du Bosquel dans la Somme après 1940. Récit, ambitions et paradoxes d'une opération singulière
}

\section{Xavier Dousson}

\section{(2) OpenEdition}

\section{Journals}

Édition électronique

URL : http://journals.openedition.org/insitu/10470

DOI : $10.4000 /$ insitu. 10470

ISSN : 1630-7305

Éditeur

Ministère de la culture

Référence électronique

Xavier Dousson, « La reconstruction du village témoin du Bosquel dans la Somme après 1940. Récit, ambitions et paradoxes d'une opération singulière », In Situ [En ligne], 21 | 2013, mis en ligne le 16 juillet 2013, consulté le 03 mai 2019. URL : http://journals.openedition.org/insitu/10470 ; DOI : 10.4000/insitu. 10470

Ce document a été généré automatiquement le 3 mai 2019.

\section{(c) $(1) \Theta \theta$}

In Situ Revues des patrimoines est mis à disposition selon les termes de la licence Creative Commons Attribution - Pas d'Utilisation Commerciale - Pas de Modification 4.0 International. 


\title{
La reconstruction du village témoin du Bosquel dans la Somme après 1940. Récit, ambitions et paradoxes d'une opération singulière
}

\author{
Xavier Dousson
}

1 Le 7 juin 1940, le village du Bosquel (240 habitants) situé à une vingtaine de kilomètres environ au sud d'Amiens, parce qu'il constitue un point de résistance à la progression de l'armée allemande, est bombardé et détruit dans sa presque totalité par cette dernière (fig. n¹). En mars 1941, sur proposition de l'architecte et urbaniste Paul Dufournet, spécialiste de l'habitat rural picard ${ }^{1}$, le Commissariat à la reconstruction immobilière choisit le Bosquel comme « village prototype ${ }^{2}$ » de réaménagement et désigne ce dernier comme urbaniste de sa reconstruction ${ }^{3}$. 
Figure 1

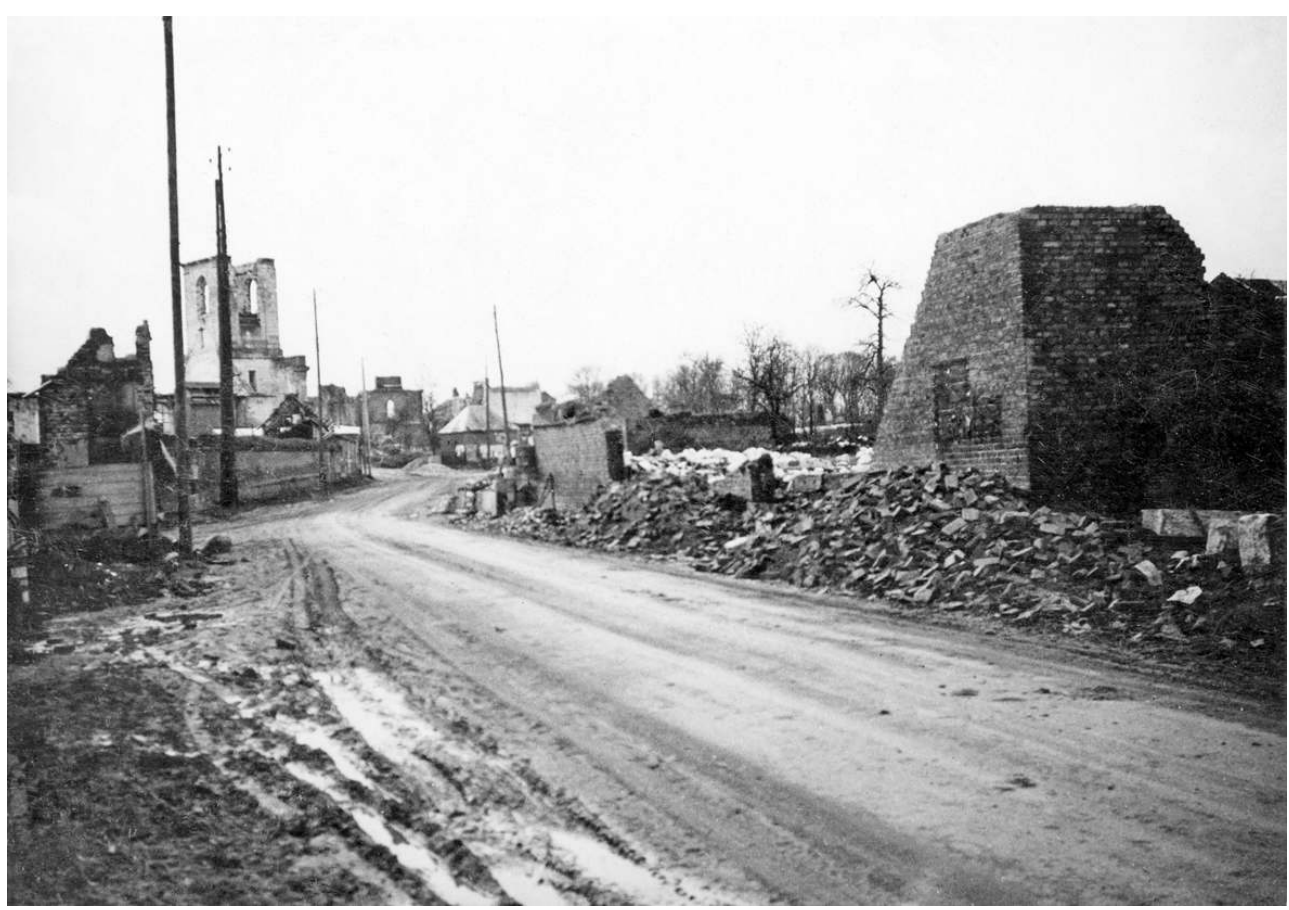

Le Bosquel au printemps 1941, ravagé par l'avancée de l'armée allemande.

(c) SIAF-CAPA - Archives d'architecture du XXe siècle, Fonds Bossu, 192 IFA 203/5.

2 En septembre, Dufournet remet un long rapport ${ }^{4}$ qui fixe les orientations générales de son projet, après avoir, dans une première partie, rédigé une monographie du village et détaillé les conséquences de sa destruction. Il y justifie en particulier son ambition d'en faire un "village d'essai» ou un "village prototype» pour plusieurs raisons liées notamment à sa situation géographique (à proximité de Paris), à la qualité de son site et à sa nature de village "purement agricole». Il insiste sur deux raisons principales. La première tient au fait que la population du Bosquel y est très " active ", "favorable aux améliorations » et a été héroïque de résistance face à l'avancée allemande. La seconde tient à l'ampleur des destructions du Bosquel qui en font une localité complètement ruinée ${ }^{5}$. Toutes ces raisons convainquent l'administration, d'abord de Vichy puis, à la Libération, de Paris, de faire du Bosquel le lieu d'une reconstruction exemplaire.

\section{La préparation de la reconstruction du « village prototype » par Paul Dufournet}

3 La tabula rasa provoquée par la guerre permet à l'urbaniste, et bientôt ses architectes d'opération, de redéfinir complètement la structuration du village, notamment la disposition de ses fonctions principales, son inscription dans l'espace rural ainsi que la nature et la forme de l'architecture de ses maisons et de ses fermes. Les principes généraux qui seront appliqués à l'aménagement du village sont définis dès ce rapport de septembre 1941 :

- remembrement complet du territoire communal ;

- « desserrement » des constructions ;

- rectification et élargissement des circulations ; 
- « dislocation » du plan traditionnel de la ferme picarde ;

- regroupement des services publics et des commerces autour des nouveaux espaces publics centraux du village. Gay. L'urbaniste en précise à diverses reprises la double originalité ${ }^{10}$ : n'avoir pas dissocié terroir et agglomération (et donc avoir pris en compte la totalité des parcelles bâties et non bâties de la commune) et avoir cherché à constituer des exploitations agricoles d'un seul tenant, sans tenir compte du statut des terres cultivées, en location ou propriété de l'exploitant.

Pour le nouveau maître d'œuvre, il faut s'attacher à concilier au mieux les nécessités de l'urbanisme et "celles d'une conception rationnelle des dispositions des bâtiments agricoles, de leur répartition et de leur aménagement ${ }^{11}$ ». Pour atteindre ce premier objectif, le territoire de la commune est théoriquement divisé en trois zones circulaires, emboîtées et complémentaires (fig. $\mathbf{n}^{\circ} 2$ ).

\section{Figure 2}
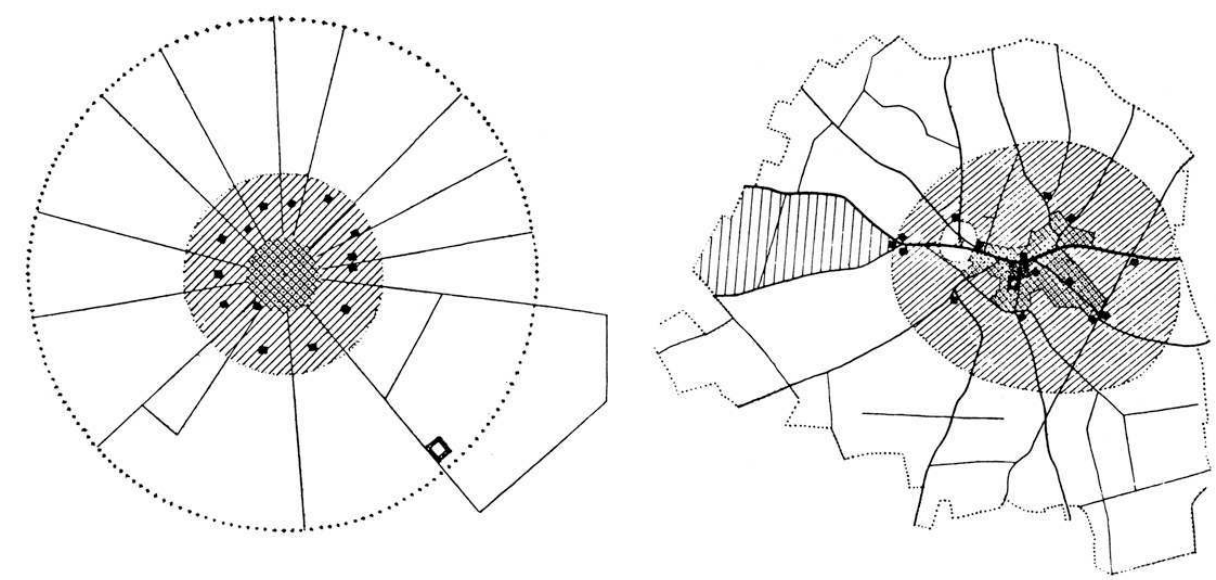

Schéma théorique. Les trois zones et le découpage du terroir en secteurs culturaux.

Au centre, la zone intérieure au périmètre d'agglomération.

Entourant ce noyau se trouve la zone d'établissements agricoles
où sont figurées les fermes des principaux cultivateur: An dela, zone dite de

Au delà, zone dite de " grande culture " comprenant tout le reste
du territoire communal. Dans l'hypothèse d'une grande ferme s'installant hors de la zone d'établissements agricoles, les services publics seraient à sa charge.

Schémas théorique et d'application de l'urbanisation du Bosquel par Paul Dufournet. DUFOURNET, Paul, en collaboration avec CALAME, François. Itinéraire en architecture rurale, Amiénois Artois Boulonnais Ponthieu (1933-1948). Lillers, Saint-Valery-sur-Somme : Sépia/Chemins de Traverses, Drac Picardie, 1991, p. 142-143.

Repro. Dousson, Xavier. (c) Xavier Dousson. 
7 Au cœur du village, la première zone accueille le centre résidentiel, commercial, culturel, cultuel et administratif. On y trouve la mairie, l'école, le foyer social, l'église et l'ensemble des commerces et locaux artisanaux disposés autour de nouveaux espaces publics, dont une grande place chère à l'urbaniste ${ }^{12}$. Autrefois fort compacte, cette zone est desserrée et aérée, en particulier grâce à l'élargissement des voies de circulation et à la nouvelle implantation des constructions qui vise à les dissocier partiellement les unes des autres. Elle est par ailleurs largement plantée, entièrement électrifiée et dotée d'une adduction d'eau municipale. L'ancien chemin départemental est promu au rang de route nationale 320.

8 Entourant ce noyau, à proximité immédiate, se trouve la zone des établissements agricoles, autrefois disséminés sur l'ensemble du territoire communal. Pour la conception de ceux-ci, Paul Dufournet affirme, peu après la Libération, dans une rhétorique presque corbuséenne ${ }^{13}$ :

«Ce n'est donc pas de formes types locales plus ou moins idéales qu'il convient de partir, mais de volume d'exploitation, de superficie cultivable, de spéculations agricoles, de commodités d'exploitation, de machinisme. La résultante: les bâtiments à volumes et dispositions conformes construits avec les matériaux les mieux qualifiés pour cet usage.

9 L'idée avancée en creux par l'urbaniste est de substituer au modèle traditionnel de la ferme picarde, issue pour lui des vicissitudes de l'histoire et de l'adaptation aux contraintes parcellaires ${ }^{14}$, des fermes fonctionnelles et rationnelles, adaptées aux nouvelles techniques d'exploitation (fig. $\mathbf{n}^{\circ} \mathbf{3}$ ). 
Figure 3

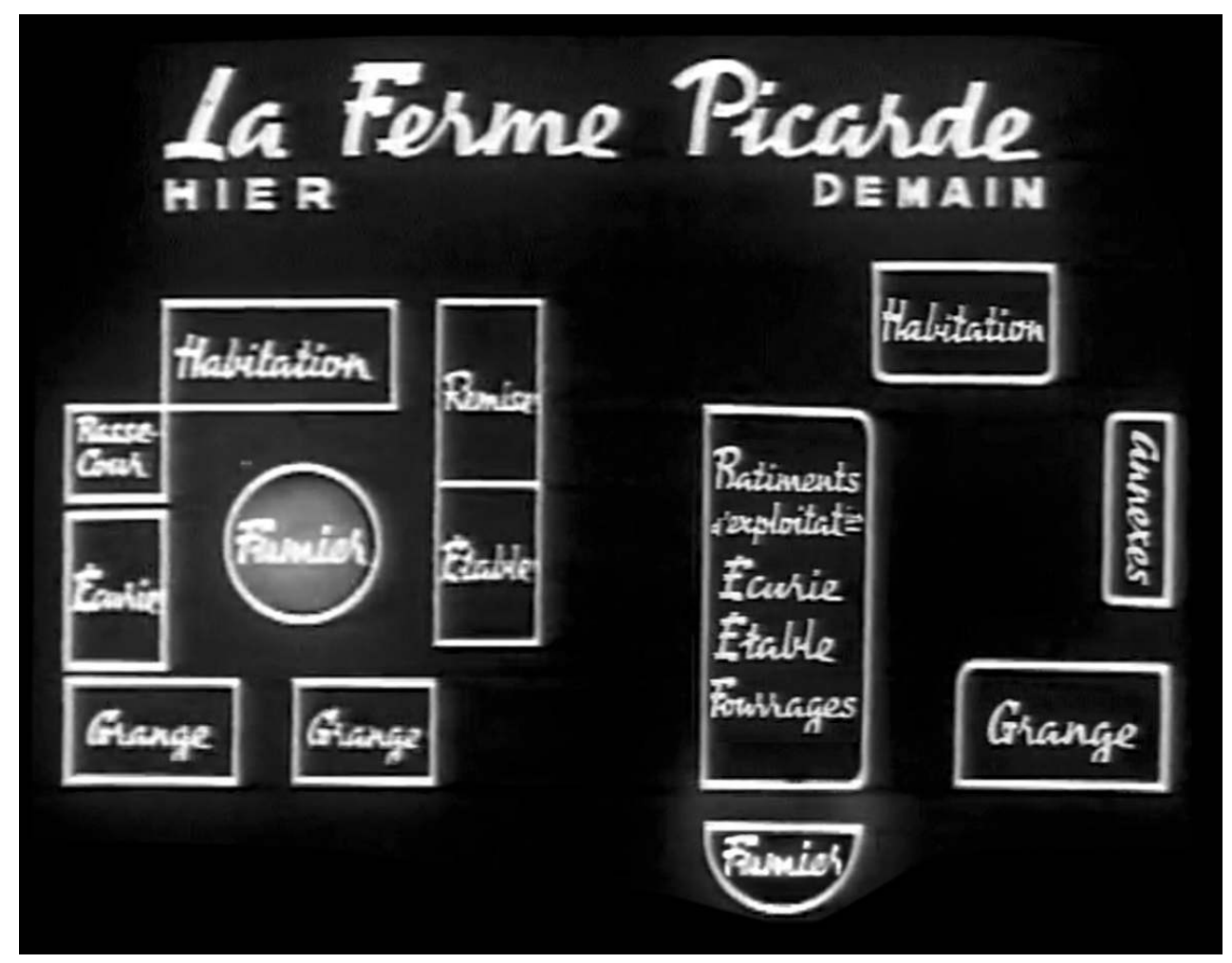

Principe d'implantation des édifices d'une ferme type du Bosquel par Paul Dufournet. Photogramme du documentaire de ROUBAIX, Paul de. Le Bosquel, un village renaît. Série «L'album cinématographique de la Reconstruction », production "Je vois tout ». Film consultable en ligne sur le site de la Cinémathèque virtuelle du Ministère de l'Egalité des territoires et du Logement et du Ministère de l'Ecologie, du Développement durable et de l'Energie. Voir note 14.

10 Au-delà de ces deux zones, s'étend ensuite la plus vaste, celle dite de " grande culture ", en prolongement direct des exploitations agricoles. Celle-ci englobe tout le reste du territoire communal et a fait naturellement l'objet des principales discussions liées au remembrement, qui a permis de diviser par presque huit le nombre des parcelles du village. Sur cette zone, les terres remembrées sont désormais redistribuées en secteurs culturaux le plus souvent de un à quatre tenants ${ }^{15}$.

11 Parallèlement à ce travail, prévoyant la pénurie de matériaux qui suivrait la fin des hostilités, Dufournet entreprend dès 1942 de faire des essais de béton de terre stabilisé aux Laboratoires du bâtiment et des travaux publics à partir d'échantillons des terres du Bosquel et des villages environnants. Ces essais seront concluants et permettront la réalisation de plusieurs édifices du village à la Libération ${ }^{16}$.

Entre 1940 et 1944, les conditions économiques difficiles ne permettent pas la reconstruction concrète du village. Cette situation d'attente va permettre la maturation d'un projet ambitieux et qualitatif, très éloigné d'une simple reconstruction à l'identique : Paul Dufournet va disposer de trois ans pour élaborer un projet radicalement différent de l'état antérieur du village, remembrer l'ensemble du territoire communal, commencer la réalisation des réseaux (adduction d'eau, électricité, etc.), préparer les esprits à son projet et anticiper les conditions matérielles de la reconstruction. Ainsi, à la Libération, le projet est si avancé qu'il peut devenir prioritaire sur la liste des opérations portées par le MRU ${ }^{17}$. 


\section{Modèle généralisable ou prototype situé ? Les ambitions d'une jeune équipe d'architectes recrutés à la Libération par Paul Dufournet} Jean Bossu, Pierre Dupré, Louis Miquel, Raymond Sénevat et Maurice Grandjean comme architectes d'opération de la reconstruction du Bosquel. Les quatre premiers sont d'anciens collaborateurs de Le Corbusier, et tous - surtout - connaissent très bien les réalités du monde agricole. Certains ont même participé aux enquêtes d'architecture rurale du musée des Arts et Traditions populaires pendant la guerre ${ }^{19}$.

Dès cette nomination, Dufournet déclare ${ }^{20}$ :

« deux principes antinomiques se présentaient à ces derniers.

Convenait-il de faire l'expérience d'un village type ?:

1) Telle qu'elle pouvait être faite avec les conceptions de l'exploitation agricole $d u$ moment, les matériaux et les moyens de réalisation disponibles pour être ensuite multipliée partout sans difficulté. Autrement dit, établir un prototype des possibilités moyennes, avec des améliorations si possible par rapport à ce qui se faisait auparavant, à condition que cela n'apporte ni gêne ni retard?

2) Ou réaliser un prototype apportant un progrès dans tous les secteurs: dispositions (tant relatives entre eux, qu'internes), des bâtiments, des systèmes de construction, etc. Soit un modèle difficile à atteindre la plupart du temps, mais qui aurait fait franchir une étape dans la façon de concevoir les habitats rural et agricole et qu'on aurait suivi ailleurs dans la mesure du possible.

C'est la première conception qu'exigeait voir appliquer M. Vaubourdolle, commissaire à la reconstruction de cette région, représentant direct du ministre. [...

] C'est la seconde conception qu'à tort ou à raison, les architectes d'opération, disciples de Le Corbusier, voulaient retenir.

D'où les retards et les heurts avec l'administration. »

L'objectif, tant pour les architectes d'opération que pour Paul Dufournet, n'est donc pas de proposer des solutions reproductibles à loisir sur l'ensemble du territoire national, avec le risque d'uniformisation que cela suppose, mais bien de créer de nouvelles particularités locales contemporaines ${ }^{21}$. Comme le souligne Dufournet, cette approche est en partie le résultat de l'influence de Le Corbusier. En effet, dans ses projets liés à l'agriculture comme celui de «ferme radieuse», celui-ci a plusieurs fois énoncé les exigences matérielles et pratiques suivant lesquelles une exploitation agricole devait être construite : "logis dignifié ", équipement moderne, silos étanches, circulation optimale, manutention mécanisée, locaux spacieux, confort et hygiène pour les bêtes, etc. ${ }^{22}$ Il a surtout donné l'esprit dans lequel cette architecture « de la terre » devait être bâtie ${ }^{23}$ :

«Une ferme n'est pas une fantaisie architecturale. C'est quelque chose de semblable à un événement naturel, quelque chose qui est comme le visage humanisé de la terre : une espèce de plante géométrique qui est aussi liée au paysage qu'un arbre ou une colline et aussi expressive de la présence humaine qu'un meuble ou qu'une machine. »

Le discours corbuséen est donc simultanément un appel à l'emploi des techniques les plus avancées pour la réalisation et l'exploitation d'un établissement agricole en même temps qu'un discours sur la nécessité « d'ancrer » la construction dans le territoire. Si la ferme est comme le "visage humanisé de la terre", semblable à un "événement naturel», celui-ci ne peut logiquement être le même partout et doit s'adapter aux conditions 
locales: terre, topographie, climat, hydrologie, cultures possibles, etc. Le discours corbuséen porte donc en lui l'idéal de relier global (solutions techniques et méthodes universelles) et local (territoire d'implantation des projets). Il implique de repenser l'agriculture à partir des données objectives du territoire et des progrès techniques ${ }^{24}$. Cet idéal, ce programme, sera repris par l'ensemble des architectes d'opération et explique, en partie, leur choix de réaliser un «village prototype » plutôt qu'un modèle généralisable ${ }^{25}$.

Pour cerner les besoins des agriculteurs, l'équipe dirigée par Paul Dufournet pousse alors très loin la concertation avec une population rurale peu habituée à être questionnée sur ses demandes en matière de cadre de vie. Cette enquête est résumée dans des comptes rendus dactylographiés ${ }^{26}$ qui détaillent avec un soin méticuleux l'ensemble des besoins et désirs des fermiers, tant pour ce qui concerne leurs exploitations que leurs habitations. $\mathrm{Au}$ sortir de la guerre, les campagnes françaises sont encore peu mécanisées et font toujours largement appel à la main-d'œuvre des ouvriers agricoles et à la force hippomobile. Par ailleurs, les exploitations ne sont pas encore «mono-orientées » vers une culture ou un élevage dominant : une exploitation agricole du Bosquel à la Libération cultive de nombreuses variétés de plantes, fruits et légumes et abrite plusieurs types d'activités provenant d'élevages différents (laiterie, bergerie, porcherie, poulailler, pigeonnier, clapiers à lapins, écuries). La situation locale est donc relativement complexe et variée : structure hiérarchisée dans les exploitations, codes sociaux, hétérogénéité des types de cultures ou d'élevage, etc. Les enquêtes révèlent cette diversité et s'attachent à détailler chaque type d'usage/action, de besoin, d'équipement ou d'espace: depuis le « circuit des pantoufles » du patron fermier jusqu'au contenu de l'armoire à pharmacie de la bergerie ! Cette précision dans l'observation et la définition des besoins va permettre aux architectes «d'ajuster » leurs projets architecturaux au plus près de la demande ${ }^{27}$. Seulement, nous y reviendrons, cet ajustement si précis sera en partie la cause de l'obsolescence fonctionnelle si rapide de ces architectures du Bosquel.

L'association entre les cinq architectes recrutés par Dufournet, auxquels s'adjoignent Robert Le Ricolais, ingénieur conseil, et Mannes Degraaf, architecte conseiller technique, est formalisée par une dénomination commune, «le service d'architecture du Bosquel »" ainsi que par un local de travail partagé, rue Saint-Roch. Cette association ne sera pourtant effective que quelques mois, le temps de définir l'ensemble des implantations des constructions du village (fig. $\left.\mathbf{n}^{\circ} \mathbf{4}\right)$ et, en particulier, le parti d'aménagement général des exploitations ${ }^{29}$, ainsi que le temps de concevoir le bâtiment d'exploitation de la ferme Quesnel. En effet, dès le début de 1946, Paul Dufournet cherche parmi son équipe un maître d'œuvre qui assume pleinement l'achèvement de la ferme Quesnel ${ }^{30}$. Ce sera Jean Bossu, qui par ailleurs en concevra et réalisera l'habitation. 
Figure 4

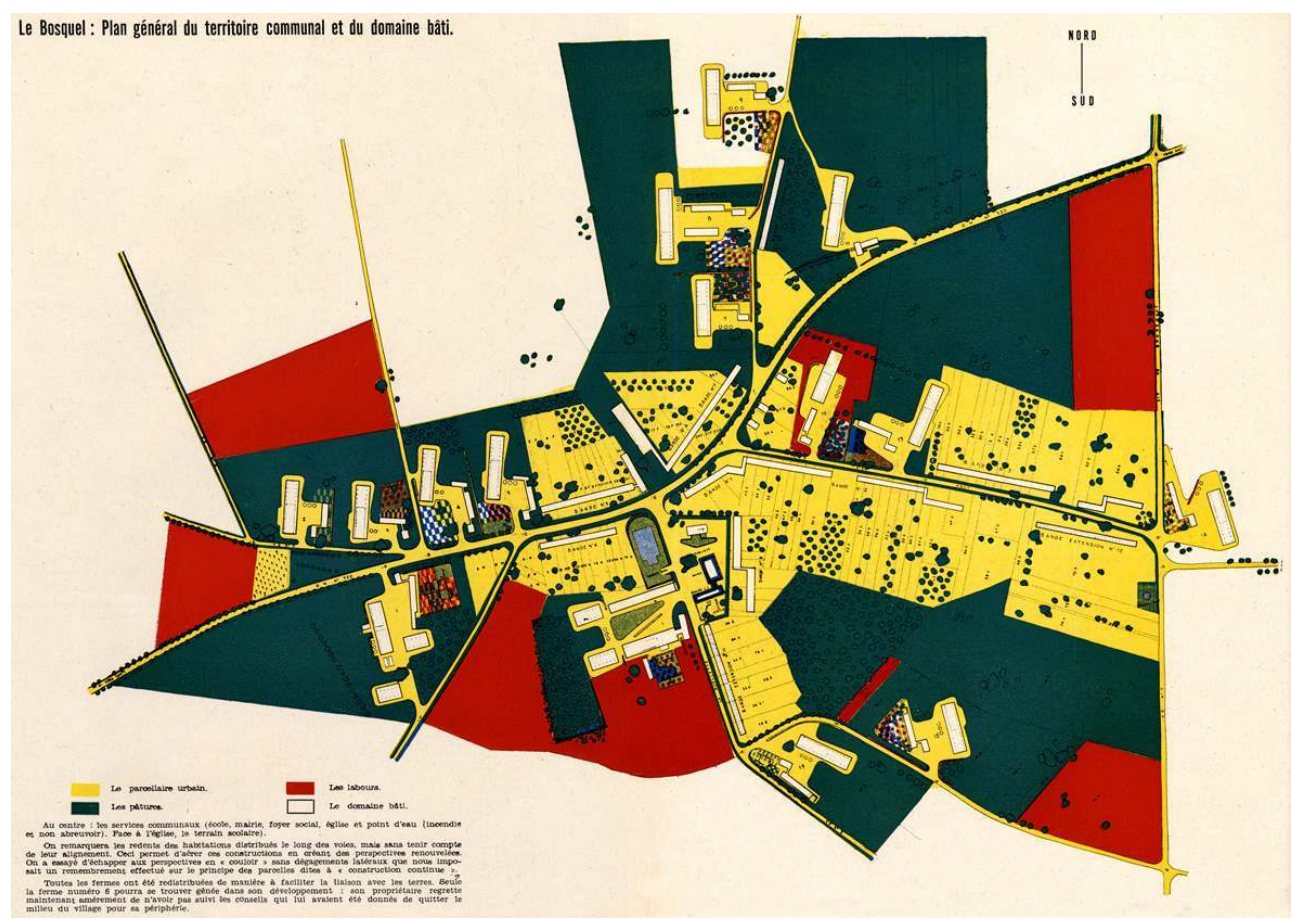

Plan d'ensemble de la reconstruction du village témoin du Bosquel, 1946. BOSSU, Jean. « Le Bosquel : préliminaires de la reconstruction et premières réalisations ». Techniques \& Architecture, 1946, nºs 3/4, p. 135-136.

Repro. Dousson, Xavier. @ Xavier Dousson.

Quelques semaines plus tard, en avril 1946, Louis Miquel démissionne de son poste et retourne à Alger exercer son métier ${ }^{31}$. Cette démission, avant même l'achèvement de la première réalisation de la reconstruction du Bosquel, n'empêchera pas l'architecte d'apparaître dans toutes les publications ultérieures de l'opération comme l'un de ses auteurs. Au contraire de Raymond Sénevat qui, bien que recruté en même temps que Miquel, n'apparaîtra dans aucune publication. Ainsi, après la lettre de Dufournet et le départ de Miquel, les autres fermes ainsi que la plupart des constructions de l'agglomération seront attribuées nominativement à chacun des architectes restants : Bossu, Dupré ou Grandjean (fig. ${ }^{\circ} 5$ ). 
Figure 5

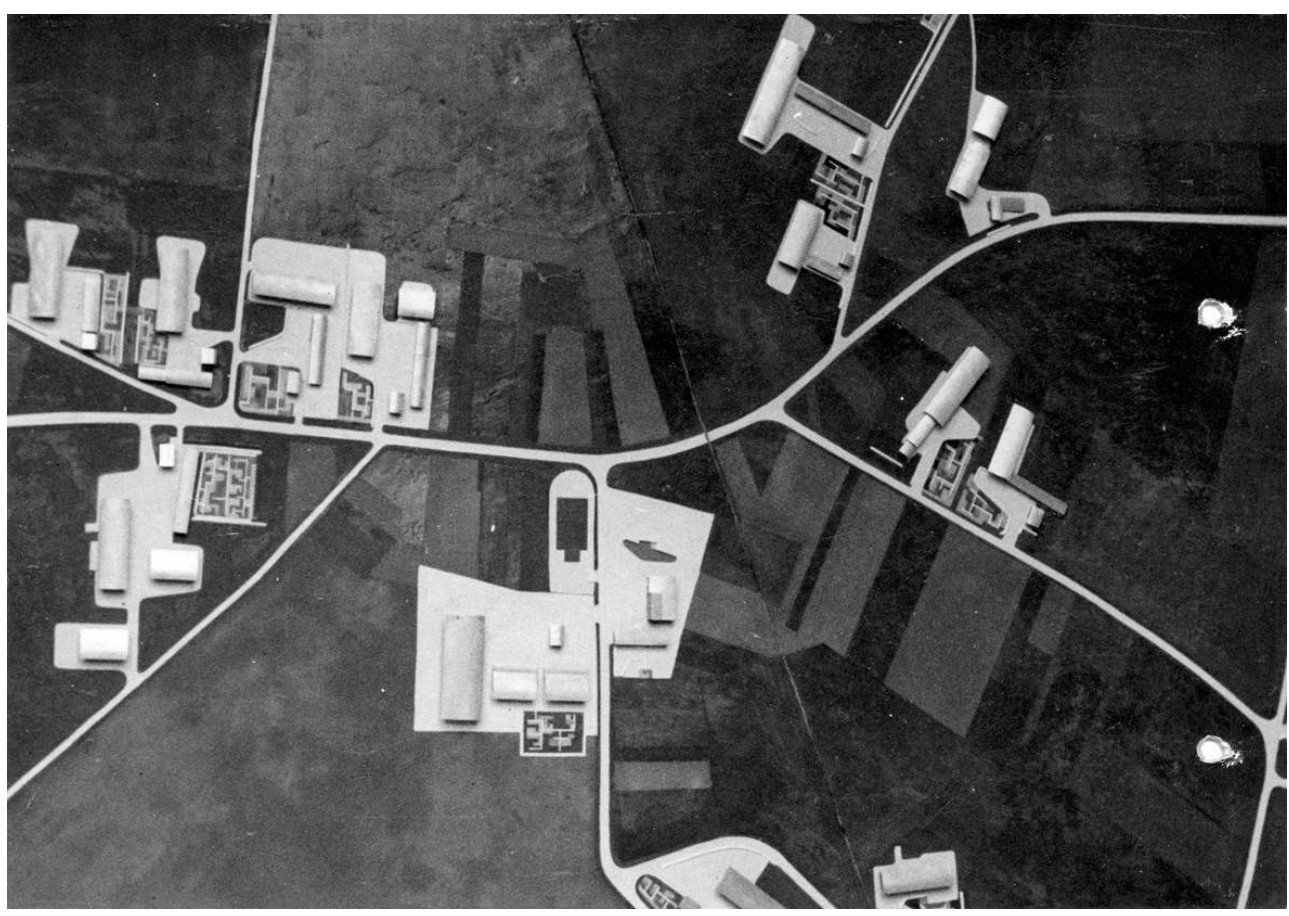

Maquette d'ensemble du projet de reconstruction du Bosquel. Claude Bachelart, Jacques Perrin et Jacques Charles, maquettistes. 1946. SIAF-CAPA - Archives d'architecture du XXe siècle - Fonds Bossu - 192 IFA 203/5.

\section{Le prototype de la ferme Quesnel}

20 Au début de 1945, le remembrement est quasiment achevé. Au mois d'août, la reconstruction du village est entamée par la réalisation de la ferme Quesnel, prototype architectural de cette reconstruction (fig. $\mathbf{n}^{\circ} \mathbf{6}$ ). Quelques mois plus tard, devant le spectacle de l'édification du bâtiment d'exploitation - le bâtiment principal de cette ferme -, les premières manifestations de mécontentement des habitants, las de vivre dans une situation provisoire depuis plusieurs années, se font jour. Dans une pétition ${ }^{32}$, ils critiquent sa massivité et redoutent son inadaptation au monde rural : " De dimensions impressionnantes, tout en béton armé, elle ressemble plus à un blockhaus pour la prochaine guerre qu'à une exploitation agricole. » 


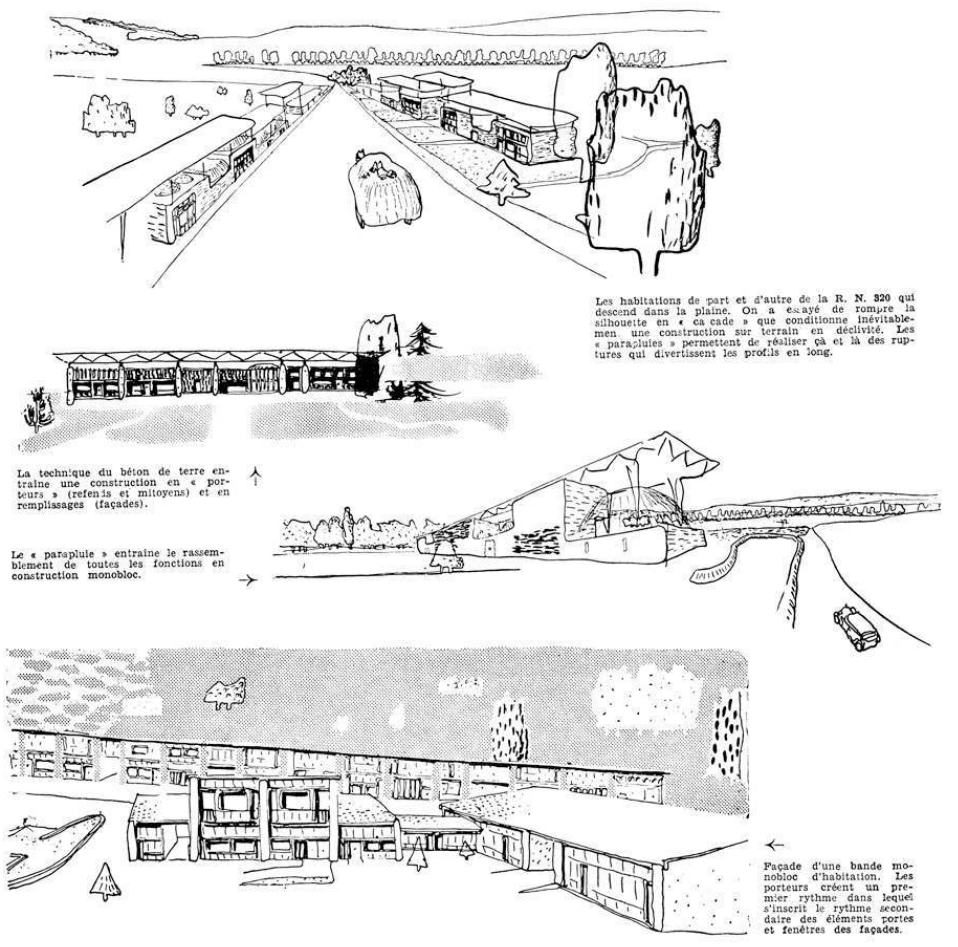

Croquis de recherches des « volumes enveloppes». Dessins de Jean Bossu. BOSSU, Jean. « Le Bosquel : préliminaires de la reconstruction et premières réalisations ». Techniques \& Architecture, 1946, nos 3/4, p. 134.

Repro. Dousson, Xavier. @ Xavier Dousson.

21 Pourtant, malgré ces protestations, la ferme Quesnel présente des dispositifs qui seront appliqués à l'ensemble des autres exploitations du village, à commencer par le schéma d'implantation de ses bâtiments qui non seulement améliore l'économie et le rendement de la ferme, mais encore en facilite le confort, l'hygiène et l'agrément. Les diverses constructions, autrefois toutes accolées, sont en effet rendues autonomes et réparties autour d'une cour désormais ouverte, mais abritée des vents grâce aux bâtiments élevés situés sur ses contours les plus exposés (fig. $\mathbf{n}^{\circ} \mathbf{7}$ ). Le fumier, cette masse malodorante, autrefois «fleuron central de la cour fermée picarde, car son importance manifestait la richesse du maître des lieux », est éloigné des maisons, repoussé à l'extrémité du bâtiment d'exploitation, en dehors d'un espace désormais libre et planté. Quatre types de bâtiments entourent celui-ci. 
Figure 7

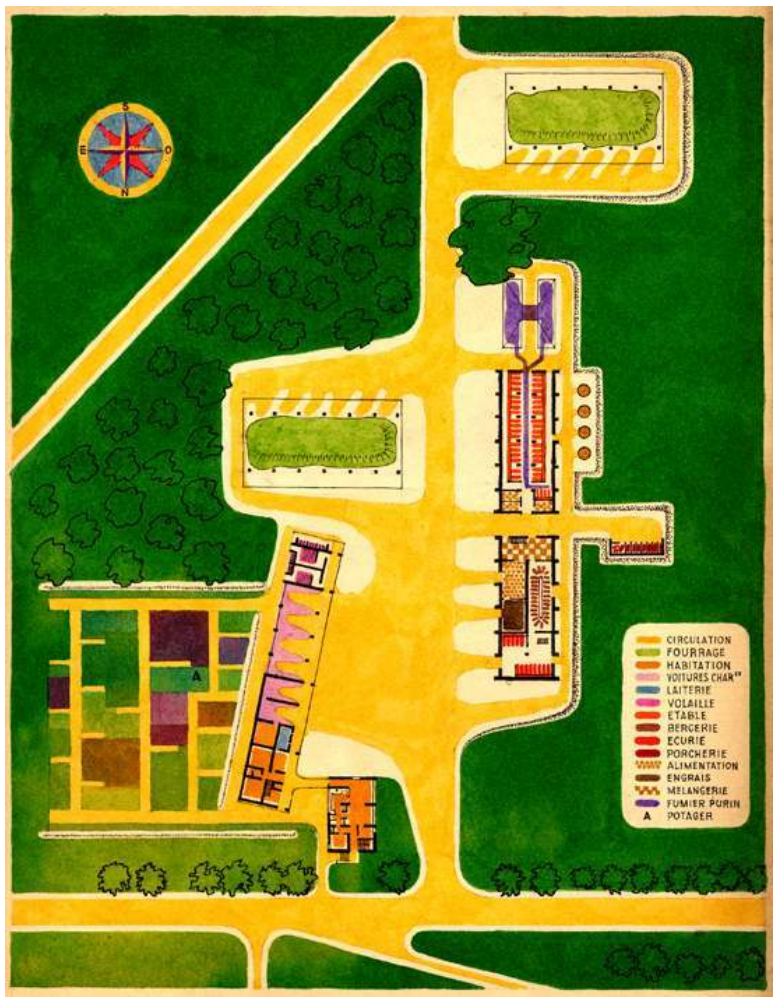

Plan d'ensemble de fonctionnement et de répartition des programmes de la ferme Quesnel. Dessin non daté.

(c) SIAF-CAPA - Archives d'architecture du XXe siècle, Fonds Bossu, 192 IFA 300/1.

Le principal bâtiment, tant par ses dimensions que par les usages qu'il permet, «l'usine agricole proprement dite » pour son architecte Jean Bossu ${ }^{33}$, est celui de l'exploitation (fig. $\left.\mathbf{n}^{\circ} \mathbf{8}\right)$. Son rez-de-chaussée abrite plusieurs types d'espaces différents (écurie, étable, bergerie, magasins à grains et à engrais, laboratoire et mélangerie) regroupés dans un «monobloc ${ }^{34}$ » de maçonnerie. Chaque espace est dimensionné en fonction de son usage et entièrement équipé, en particulier de meubles en béton moulé (mangeoires, auges, abreuvoirs). 


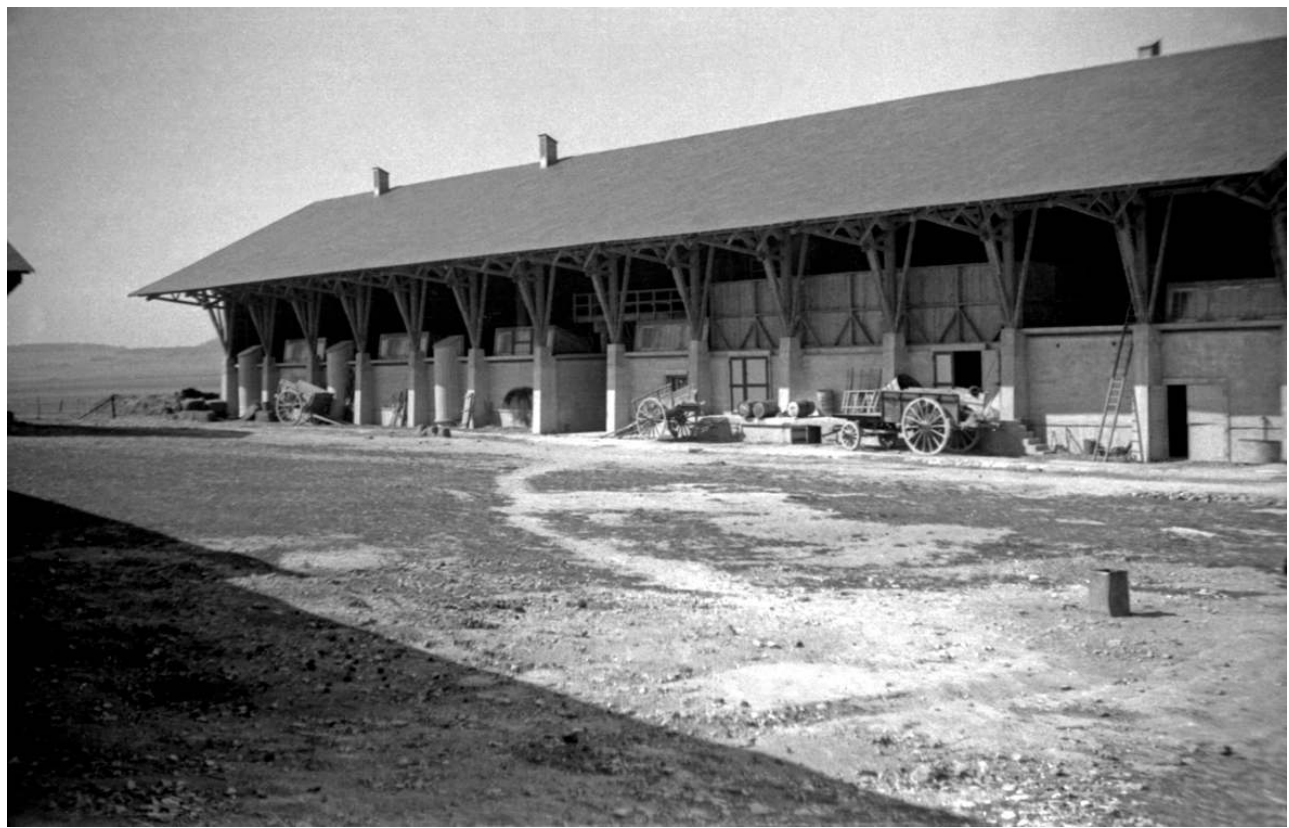

Le bâtiment d'exploitation de la ferme Quesnel vu depuis la cour. Vers 1947.

(c) SIAF-CAPA - Archives d'architecture du XXe siècle, Fonds Bossu, 192 IFA 203/14.

23 L'édifice de 65 mètres de long environ et de treize travées est ouvert en son centre par un passage de la largeur d'une travée entière mettant directement en relation les terres cultivées et la cour de la ferme (fig. $\mathbf{n}^{\circ} \mathbf{9}$ ). Tous les espaces réservés aux animaux ouvrent de plain-pied sur les pâtures et les terres par de vastes portes faciles à manipuler. Des caves à betterave et à pomme de terre sont réalisées en sous-sol. Elles remplacent les anciens silos qui disparaissent ainsi du paysage du village. Enfin, à l'étage, sous le vaste faîte de la charpente en bois, dont le large auvent permet d'accroître la surface de protection, les architectes installent le fenil, les greniers à céréales ensachées ainsi qu'une chambre pour surveiller les bêtes. 
Figure 9

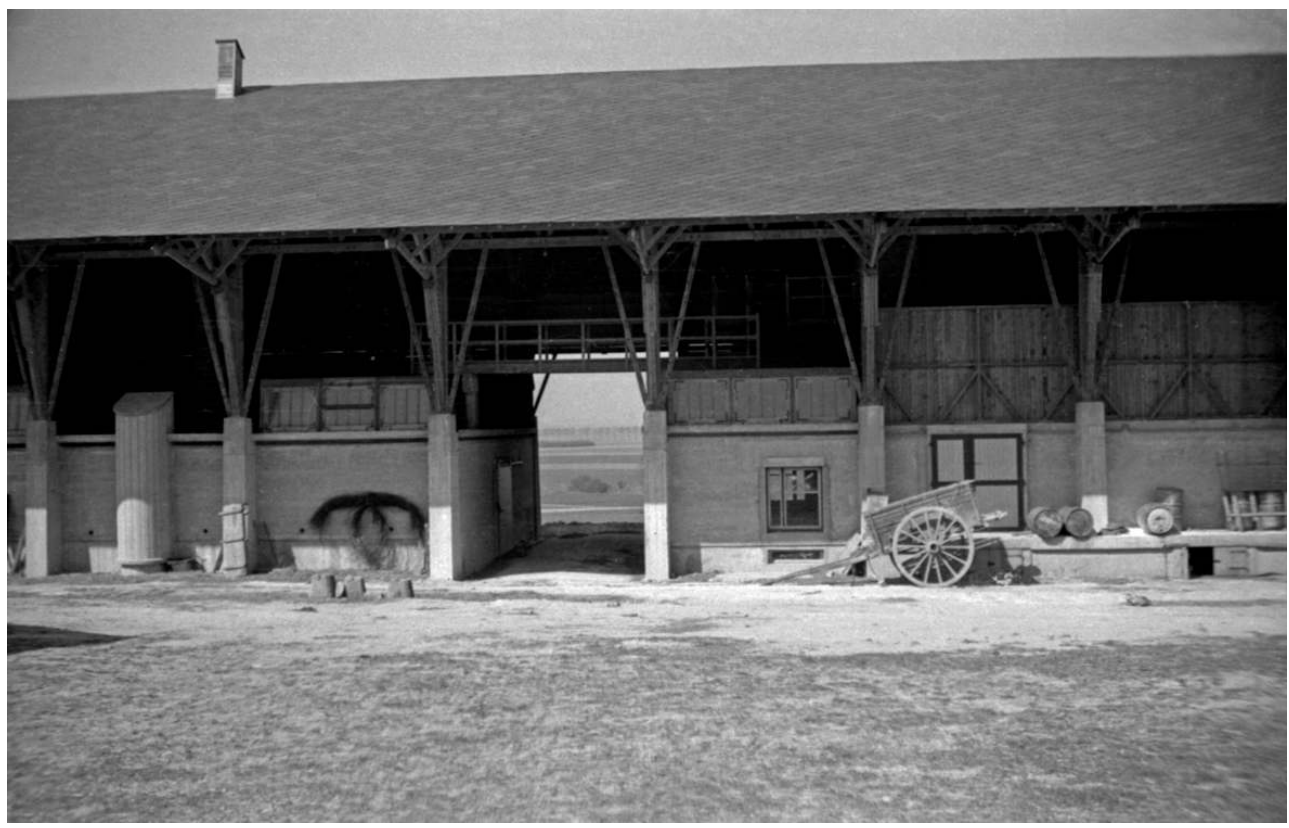

Bâtiment d'exploitation de la ferme Quesnel : vue du passage au travers de l'édifice ouvrant sur les terres cultivées, depuis la cour. Vers 1947.

(c) SIAF-CAPA - Archives d'architecture du XXe siècle, Fonds Bossu, 192 IFA 203/14.

24 Les architectes distinguent donc explicitement le rez-de-chaussée en maçonnerie de l'étage, partiellement ouvert, en charpente bois. La trame constructive est laissée apparente, elle exprime la rationalité de la construction. Les remplissages sont distincts de celle-ci, tout comme les percements, ainsi que les systèmes techniques d'alimentation du bétail - « les abats-foin ${ }^{35} »-$, disposés en fonction des nécessités d'usage (fig. $\left.\mathbf{n}^{\circ} \mathbf{1 0}\right)$. 
Figure 10

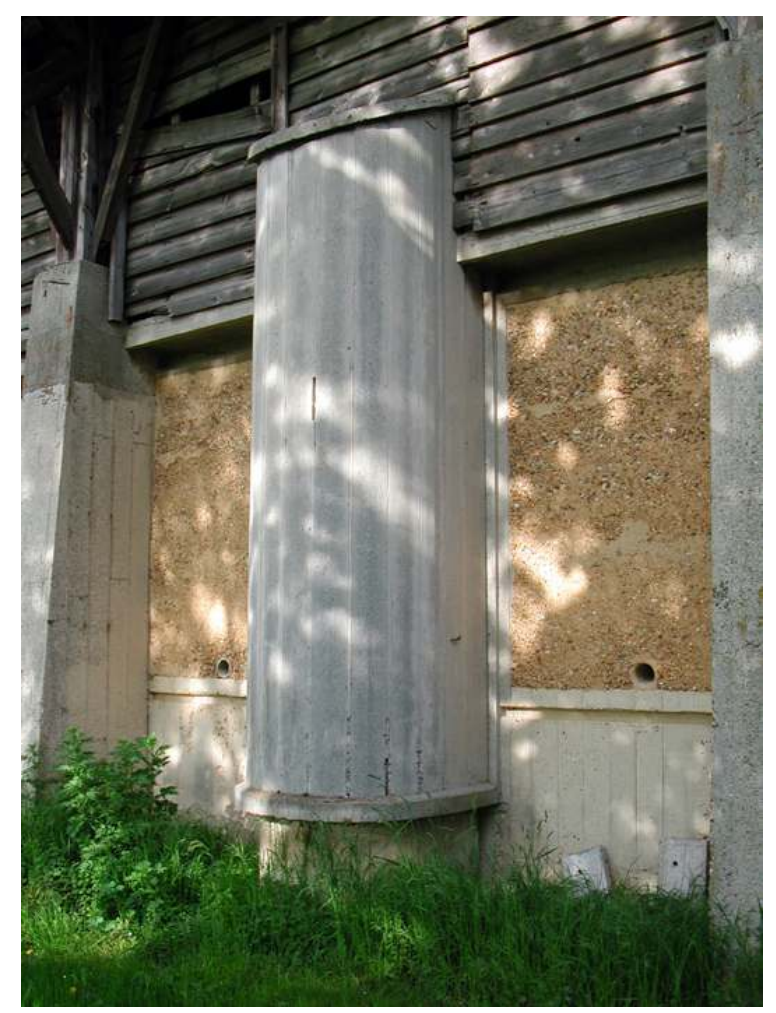

Détail d'un « abat-foin » du bâtiment d'exploitation de la ferme Quesnel. Vue contemporaine. 20 mai 2002.

Phot. Dousson, Xavier. (c) Xavier Dousson.

Le deuxième bâtiment construit est celui des annexes de l'exploitation (fig. $\mathbf{n}^{\circ} \mathbf{1 1}$ ). Disposé à proximité de l'habitation, il abrite garages, charretterie, atelier, poulaillers, clapiers, buanderie, laverie, laiterie, bûcher, quelques chambres de domestiques et des douches pour le personnel. Ces différents programmes sont contenus dans plusieurs volumes, accolés ensemble de manière à former une sorte de bloc unitaire. Néanmoins, ils se distinguent les uns des autres par le dessin des murs de refend en béton de terre, tous différents et parfois décalés, les percements et remplissages ainsi que par les types et les hauteurs de couvertures. Tous ces volumes sont reliés entre eux par un cheminement sur la façade ouest, découpé par des baies en arc évidées dans la maçonnerie. 
Figure 11

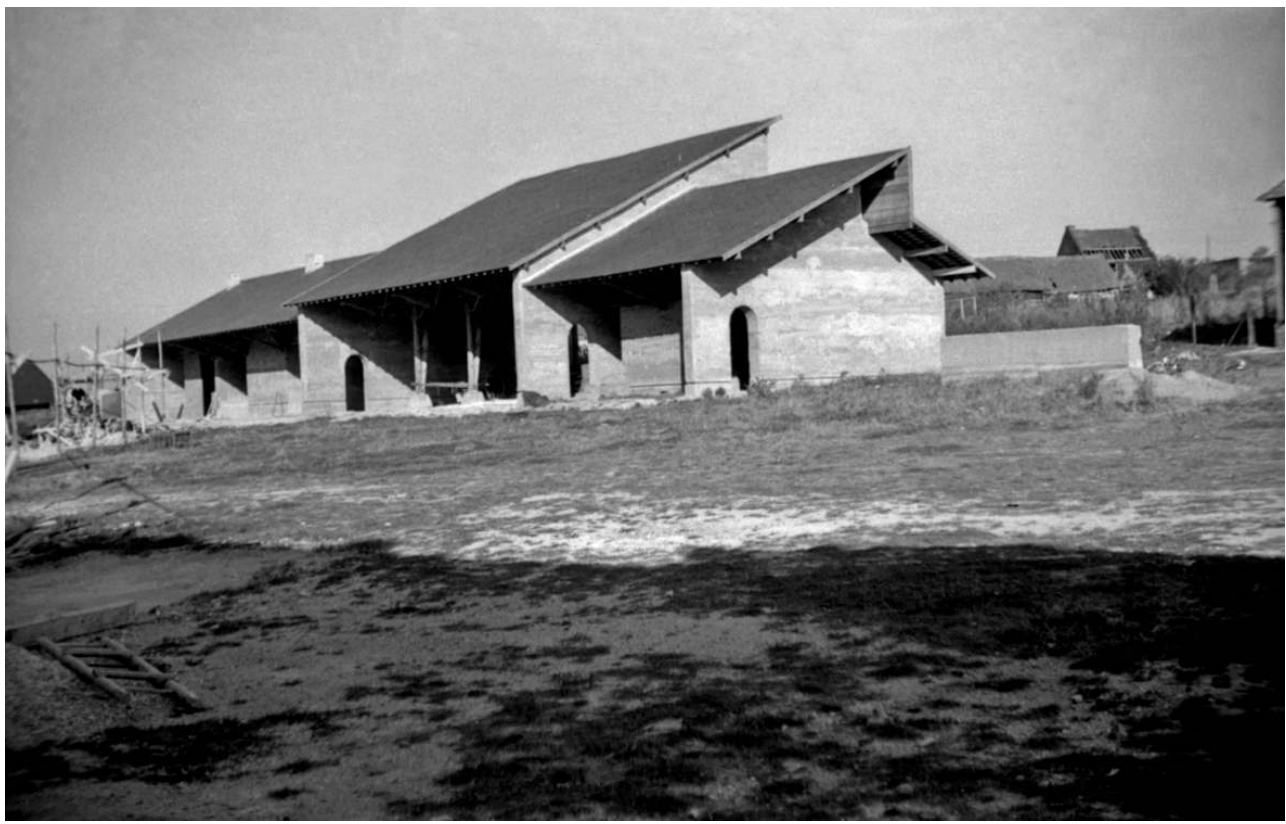

Le bâtiment des annexes de la ferme Quesnel vu depuis la cour. Mai 1947.

(c) SIAF-CAPA - Archives d'architecture du XXe siècle, Fonds Bossu, 192 IFA 203/14.

Le troisième bâtiment est un hangar à gerbier (meules) et matériel divers conçu comme un vaste auvent dont la charpente en bois est construite sur le même modèle que celle du bâtiment d'exploitation. Il vient refermer la cour au sud (fig. $\mathbf{n}^{\circ} \mathbf{1 2}$ ).

Figure 12

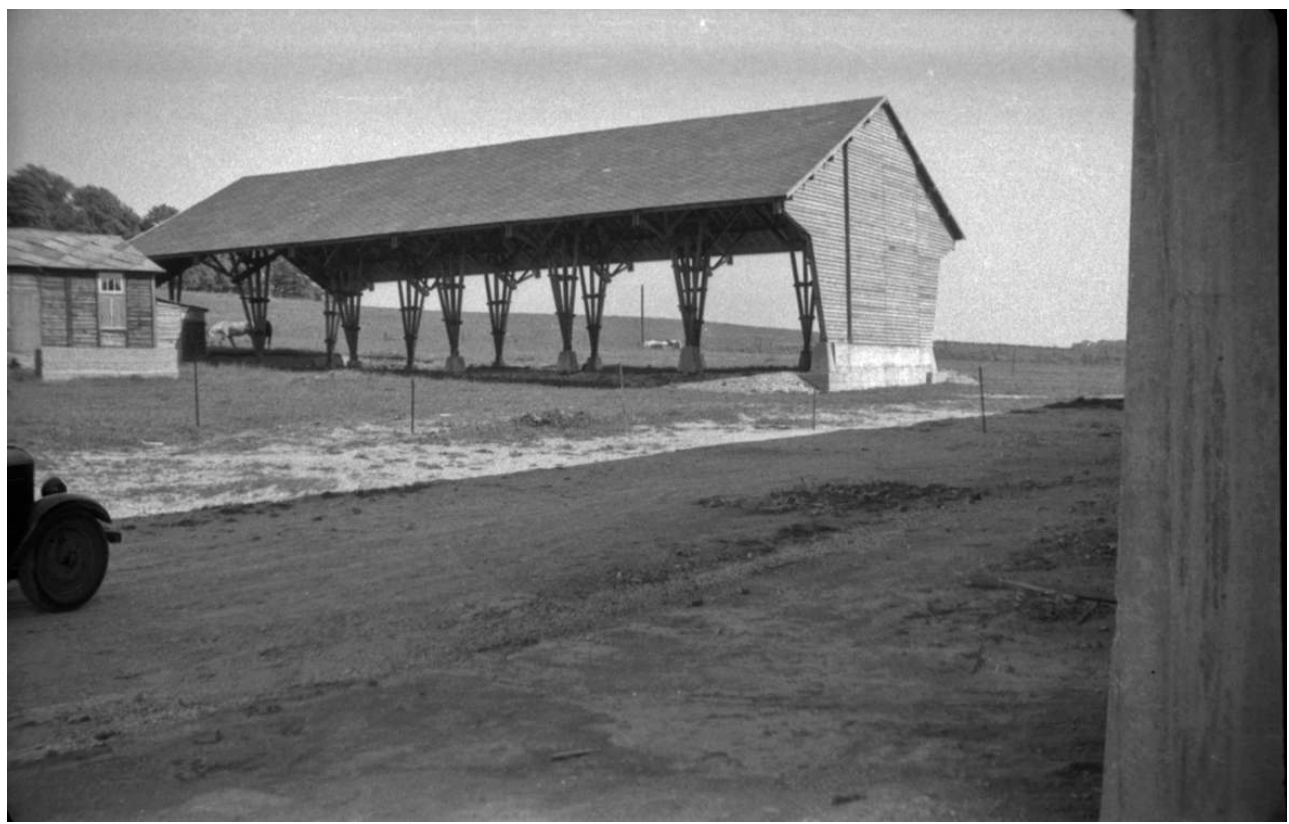

Le hangar de la ferme Quesnel vu depuis la cour.

(c) SIAF-CAPA - Archives d'architecture du XXe siècle, Fonds Bossu, 192 IFA 203/14. 
L'habitation du fermier est la dernière réalisation de la reconstruction de la ferme Quesnel (fig. n¹3). Entièrement dessinée par Bossu et ses collaborateurs, elle est située au bord de la route afin de faciliter la surveillance de l'entrée de l'exploitation et les rapports avec le village. Commandant la cour, légèrement surélevée, elle est implantée assez loin des animaux et des manutentions bruyantes et poussiéreuses, à l'opposé du fumier. À l'intérieur, les locaux de travail et l'appartement privé sont nettement différenciés jusqu'à posséder des entrées distinctes. D'autres dispositifs, nombreux et inhabituels dans la région, doivent dans l'esprit de l'architecte en faciliter l'usage, alléger le travail et favoriser l'hygiène. Des couloirs et portes sont ainsi dessinés pour éviter que les pièces ne se commandent. Un bureau est prévu pour l'exploitant. Un lavabo et un portemanteau sont installés dans l'entrée de service des ouvriers agricoles. Une cloison mobile est prévue pour séparer la salle commune de la salle à manger privée ainsi qu'un meuble séparatif avec passe-plat entre la cuisine et la salle commune. La lumière naturelle est dispensée dans toutes les pièces par de nombreuses fenêtres et impostes vitrées. Les chambres sont à l'étage. Elles ouvrent toutes sur la plaine à l'ouest et sont accompagnées d'une salle de bains. Elles sont desservies par un large couloir bordé d'un long placard.

Figure 13

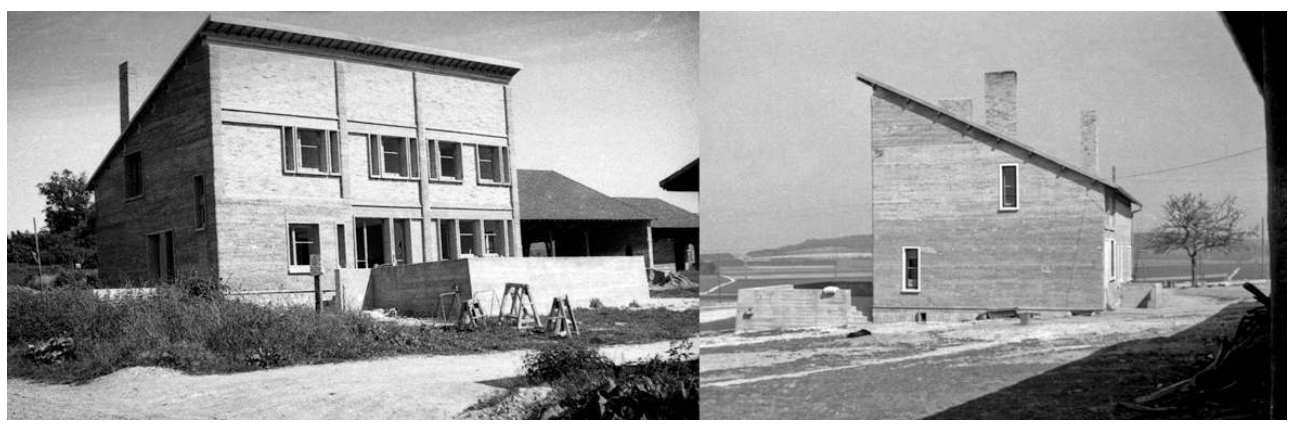

Le bâtiment d'habitation de la ferme Quesnel : façade ouest et pignon sud. Mai 1947.

(c) SIAF-CAPA - Archives d'architecture du XXe siècle, Fonds Bossu, 192 IFA 203/14.

Cette maison, entièrement composée avec l'aide du modulor ${ }^{36}$, tranche par son originalité (fig. $\mathbf{n}^{\circ} \mathbf{1 4}$ ), non seulement par rapport aux habitations antérieures, mais également par rapport à celles qui lui seront postérieures. Baptisée « ferme-cobaye » par un journaliste ${ }^{37}$ , elle se distingue clairement des autres constructions par une toiture à une seule pente, des murs de refend en béton de terre stabilisée et une vaste terrasse de béton dans le prolongement du séjour. Son volume est ainsi orienté vers la cour et la plaine ${ }^{38}$. La trame des murs de refend en béton de terre et des planchers en béton armé est «libre» et apparente. Les remplissages et les percements sont distincts de la construction: légèrement en retrait, disposés librement en fonction des nécessités et traités avec une variété de matériaux différents de la structure (briques, enduit, cadres de béton peints, verre). Les percements des pignons sont ponctuels, réduits et soulignés de blanc. 
Figure 14

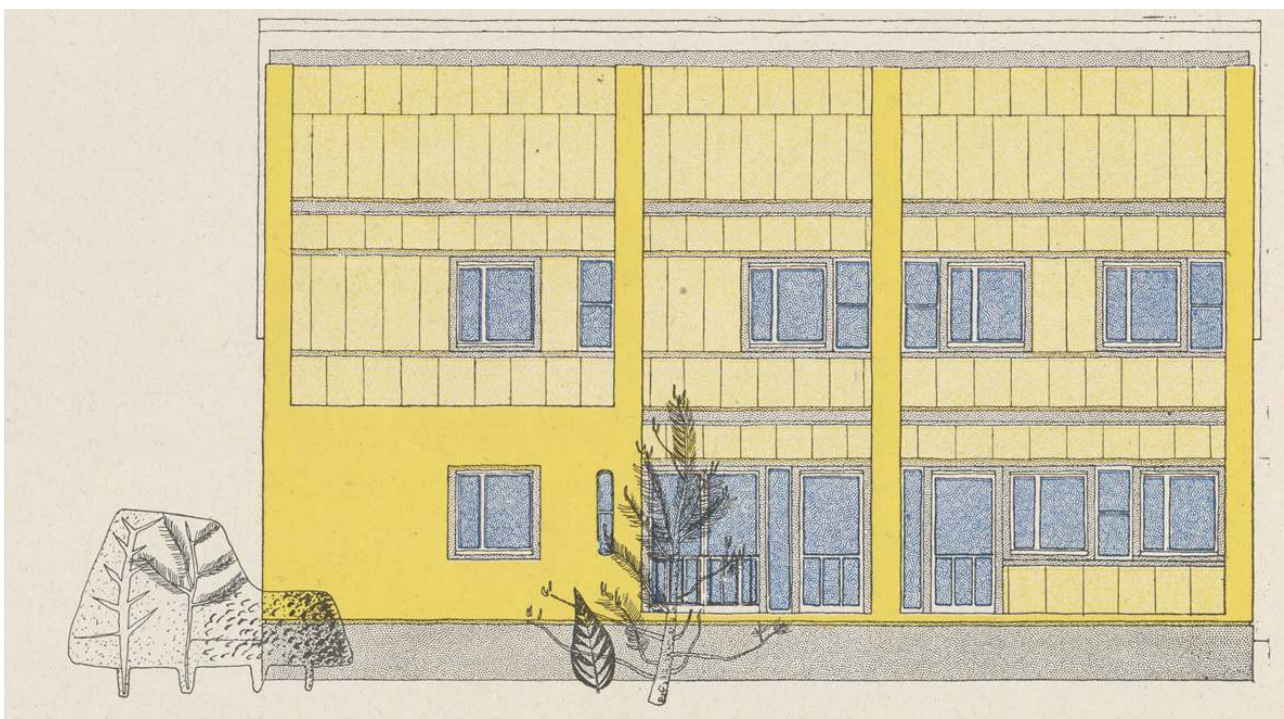

Élévation de la façade ouest du bâtiment d'habitation de la ferme Quesnel. BOSSU, Jean. «Le Bosquel : préliminaires de la reconstruction et premières réalisations ». Techniques \& Architecture, 1946, nos 3/4, p. 141.

Repro. Dousson, Xavier. (c) Xavier Dousson.

Toutes les autres fermes du Bosquel seront réalisées sur la base du modèle de la ferme Quesnel, rationalisé, simplifié et adapté aux divers autres exploitants (fig. $\mathbf{n}^{\mathbf{0}} \mathbf{1 5}$ ). Néanmoins le prototype, par la richesse de ses dispositifs, l'utilisation généralisée du béton de terre, son importance (elle est la deuxième ferme du village en taille) et son état de conservation reste l'élément emblématique de cette reconstruction ${ }^{39}$.

\section{Figure 15}

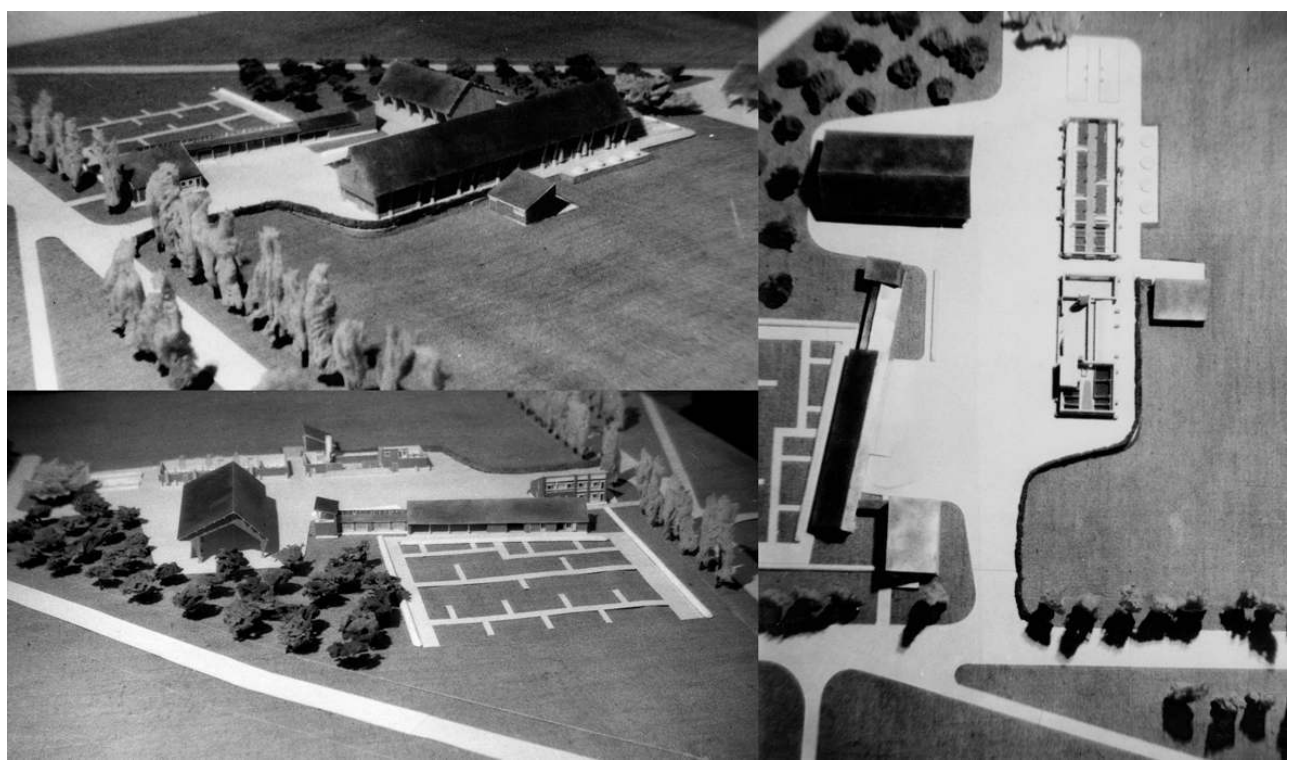

Vues de la maquette de la ferme Quesnel. Fabien Vienne et Mannes Degraaf, maquettistes.

(c) SIAF-CAPA - Archives d'architecture du XXe siècle, Fonds Bossu, 192 IFA 203/5; 192 ifa 203/14. 


\section{L'invention et l'expérimentation de techniques constructives}

La reconstruction du Bosquel est également le terrain de nombreuses inventions et expérimentations techniques, en particulier l'étude de charpentes tridimensionnelles par Le Ricolais (finalement abandonnées), l'utilisation du béton de terre pour la réalisation de plusieurs constructions, la fabrication de parpaings de briquaillons et un travail poussé sur la préfabrication légère et manutentionnable. Très loin d'être anecdotique, cette dimension matérielle et technique du projet va considérablement marquer son aspect plastique, architectural, et participer pleinement à l'objectif des architectes d'offrir au village une architecture exprimant et découlant de ses particularités locales.

L'élément peut être le plus spectaculaire des recherches conduites au Bosquel est le travail mené par Robert Le Ricolais pour la définition du système de couverture des bâtiments d'exploitation et des hangars (fig. $\mathbf{n}^{\circ} \mathbf{1 6}$ ). L’ingénieur étudie en effet pour cela un système révolutionnaire de charpentes tridimensionnelles - le système Aplex-, présenté dès la première publication du projet dans Techniques \& Architecture, en $1946^{40}$. [LIEN SUR ARTICLE CIVIDINO A FAIRE]

Figure 16

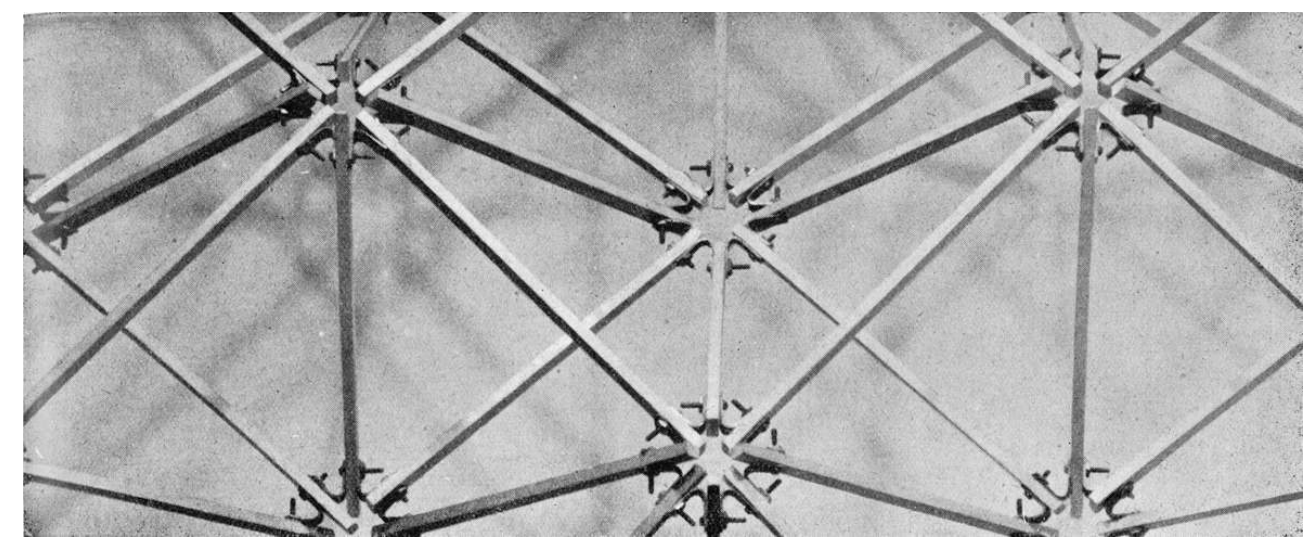

Structure tridimensionnelle étudiée par Robert Le Ricolais pour les exploitations agricoles du Bosquel. BOSSU, Jean. «Le Bosquel : préliminaires de la reconstruction et premières réalisations ». Techniques \& Architecture, 1946, nos 3/4, p. 131.

Repro. Dousson, Xavier. (C) Xavier Dousson.

Malgré ses qualités économiques et plastiques (les «parapluies » dessinés par Bossu pour les publications), ce système ne sera pourtant pas utilisé (fig. $\mathbf{n}^{\circ}{ }^{17}$ ). Paul Dufournet en donne, avec un brin d'amertume, l'explication ${ }^{41}$ :

«Un système excellent, très remarquable d'effet, et économique de surcrôit, mis au point par l'ingénieur Le Ricolais avait été proposé. Il s'agissait de fermes de charpente en bois à éléments réticulés sur le principe des radiolaires. Il fut refusé par le commissaire à la Reconstruction sous prétexte qu'on ne pouvait pas fermer le Crémona ${ }^{42}$ autrement dit, qu'on ne pouvait pas le calculer. » 

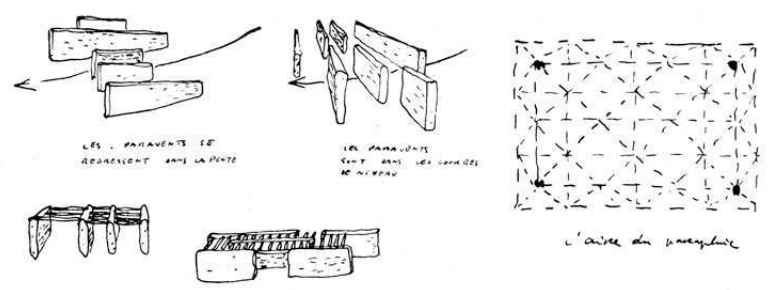

REC HERCHES

DE STRUCTURES
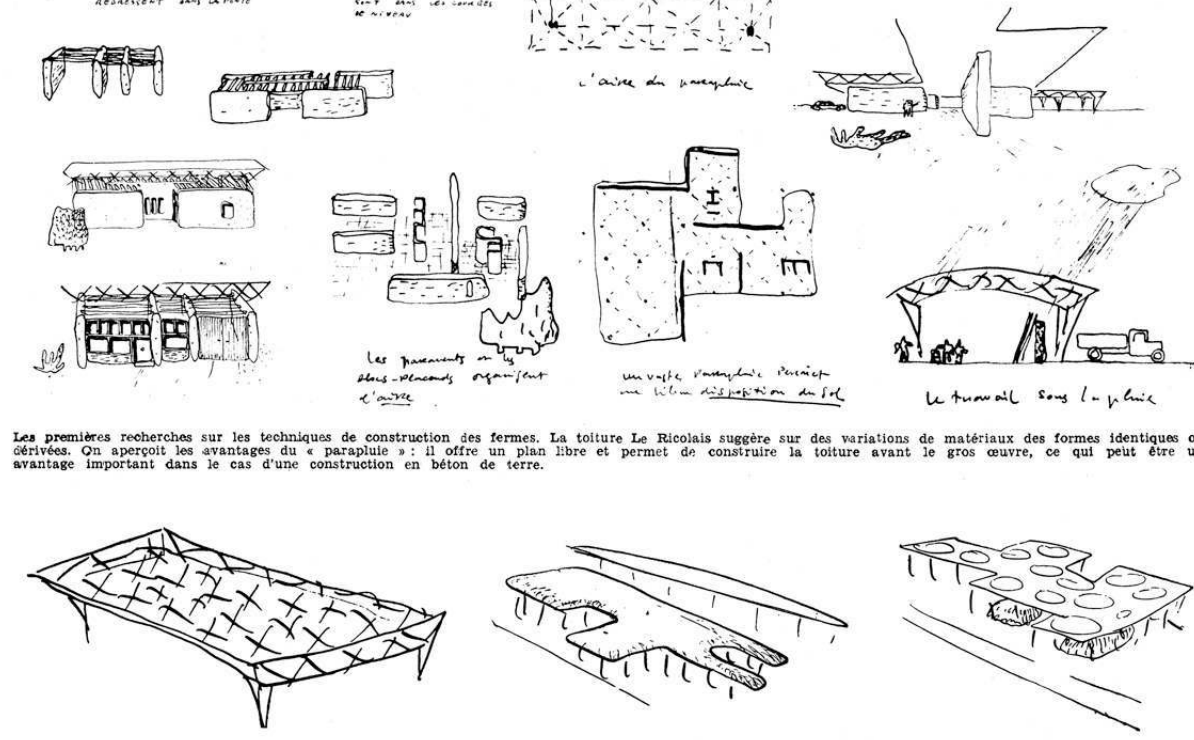

Le principe de construction du Bosquel portait sur ce thè
das contenants reallses en dur (logement des bêtes, etc...)

Croquis des « recherches de structures ». Dessins de Jean Bossu. BOSSU, Jean. «Le Bosquel : préliminaires de la reconstruction et premières réalisations ». Techniques \& Architecture, 1946, nos 3/4, p. 133.

Repro. Dousson, Xavier. @ Xavier Dousson.

C'est finalement une charpente en bois étudiée par l'ingénieur Gauthier ${ }^{43}$ qui sera réalisée pour le bâtiment d'exploitation et pour le hangar de la ferme Quesnel (fig. $\left.\mathbf{n}^{\circ} \mathbf{1 8}\right)$. Ce type de charpente sera ensuite repris, à quelques variations dimensionnelles près, pour la réalisation de pratiquement toutes les autres exploitations du village $e^{44}$. Les charpentes dessinées par Gauthier, si elles ne présentent pas le même intérêt historique que celles qu'aurait pu réaliser Le Ricolais ${ }^{45}$, sont néanmoins d'un raffinement constructif et d'une solidité exemplaires ${ }^{46}$. L'apport de Le Ricolais, présenté dans l'ensemble des publications du projet comme l'ingénieur conseil de la reconstruction du Bosquel, au contraire de Gauthier moins souvent cité, reste finalement mal documentée ${ }^{47}$. 


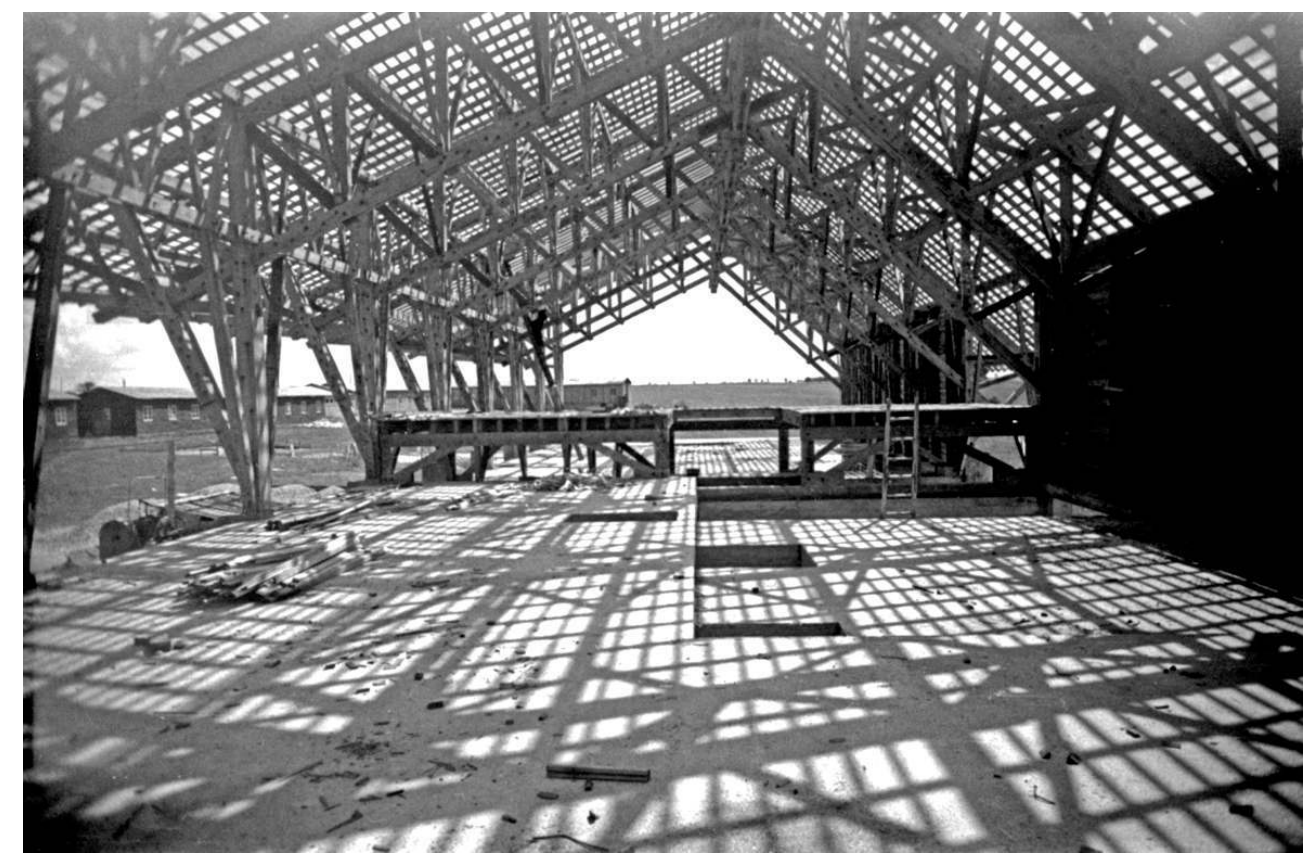

La charpente du bâtiment d'exploitation de la ferme Quesnel en cours de construction. Cliché non daté, vers 1947.

(c) SIAF-CAPA - Archives d'architecture du XXe siècle, Fonds Bossu, 192 IFA 203/14.

La reconstruction du Bosquel est également l'occasion d'utiliser à quatre reprises la technique du béton de terre stabilisée, soit en éléments de remplissage, soit comme structure porteuse. Ainsi, les murs de remplissage du «monobloc» du bâtiment d'exploitation de la ferme Quesnel sont en béton de terre stabilisée, tout comme les murs de refend porteurs de l'habitation et des annexes de cette même ferme. Par ailleurs, l'habitation de la ferme Dupont (une exploitation de 60 hectares) est également édifiée en béton de terre.

Son utilisation est justifiée à l'époque du fait de la pénurie de matériaux : il suffit en effet d'environ 100 kilogrammes de ciment seulement pour faire un mètre cube de béton de terre stabilisée ${ }^{48}$. Moins résistants qu'une solution identique en béton armé, les murs en béton de terre doivent être édifiés en respectant une épaisseur de 40 à 50 centimètres environ, ce qui a pour avantage de les rendre particulièrement résistants aux intempéries et isothermes. Le coût économique d'un mur en béton de terre stabilisée est comparable à celui d'un mur de brique et légèrement supérieur à celui d'un mur en aggloméré de sable et ciment de 30 centimètres d'épaisseur (fig. $\mathbf{n}^{\circ} \mathbf{1 9}$ ). 


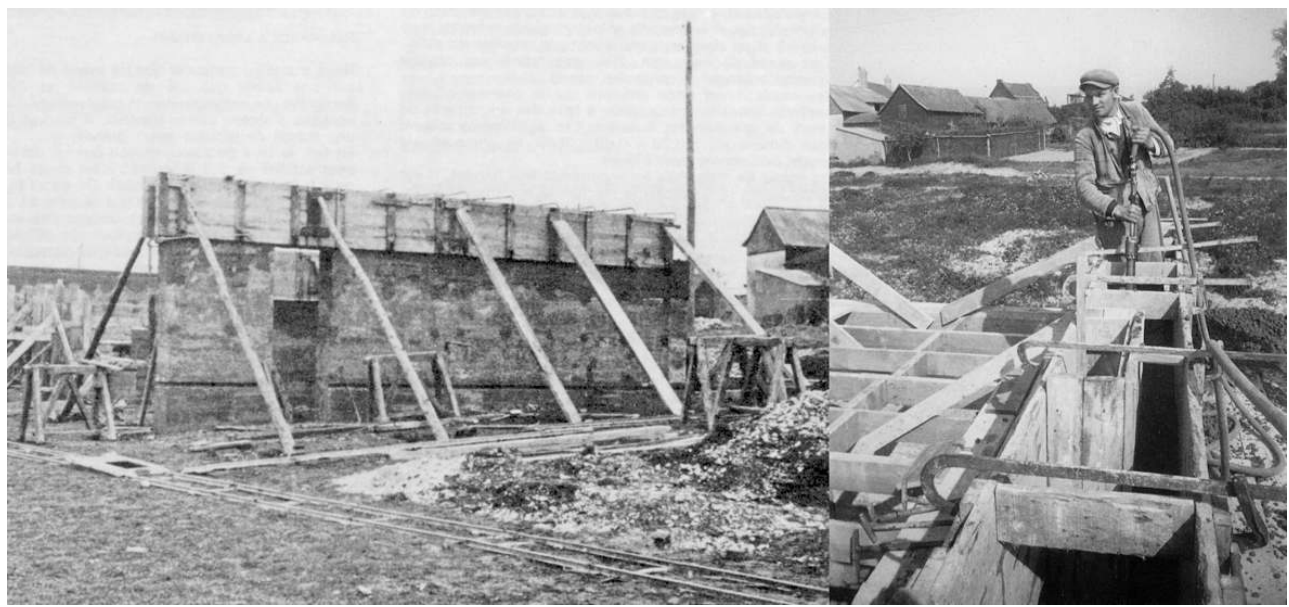

Mise en œuvre du béton de terre des annexes de la ferme Quesnel. DUFOURNET, Paul, en collaboration avec CALAME, François. Itinéraire en architecture rurale, Amiénois Artois Boulonnais Ponthieu (1933-1948). Lillers, Saint-Valery-sur-Somme : Sépia/Chemins de Traverses, Drac Picardie, 1991, p. 155 et 174

Repro. Dousson, Xavier. (C) Xavier Dousson.

Au-delà de ces considérations économiques et techniques, le béton de terre stabilisé possède, d'un point de vue plastique, des qualités remarquables qui n'échappent pas à Paul Dufournet ${ }^{49}$ :

«Formes larges et grasses qui donnent une impression de densité et de force. Parement grenu, suffisamment irrégulier pour n'être pas monotone, d'aspect vivant comme une matière organique. Quelquefois, la superposition des couches pilonnées suggère une stratigraphie rocheuse.

Belle couleur chaude de pain bis. Le bâtiment prend sa robe quelques mois après sa finition. Les ragréments se fondent dans la masse générale. »

Cette citation de 1950 pourrait aussi bien être contemporaine, tant ce matériau conserve intactes ses propriétés plastiques et constructives dans le temps, et ceci quasiment sans entretien (fig. $\mathbf{n}^{\circ} \mathbf{2 0}$ ). Pourtant son expérimentation sera arrêtée, essentiellement pour des raisons psychologiques, du fait de sa trop grande parenté avec le pisé des fermes antérieures, synonyme de fragilité et de non-durabilité pour les habitants. De nos jours, le regain des recherches et études sur l'architecture en pisé ou en béton de terre ${ }^{50}$, porté en particulier par les enjeux environnementaux, tout comme l'utilisation de ce matériau par plusieurs architectes contemporains éminents ${ }^{51}$, montre la pertinence de ce choix constructif. 


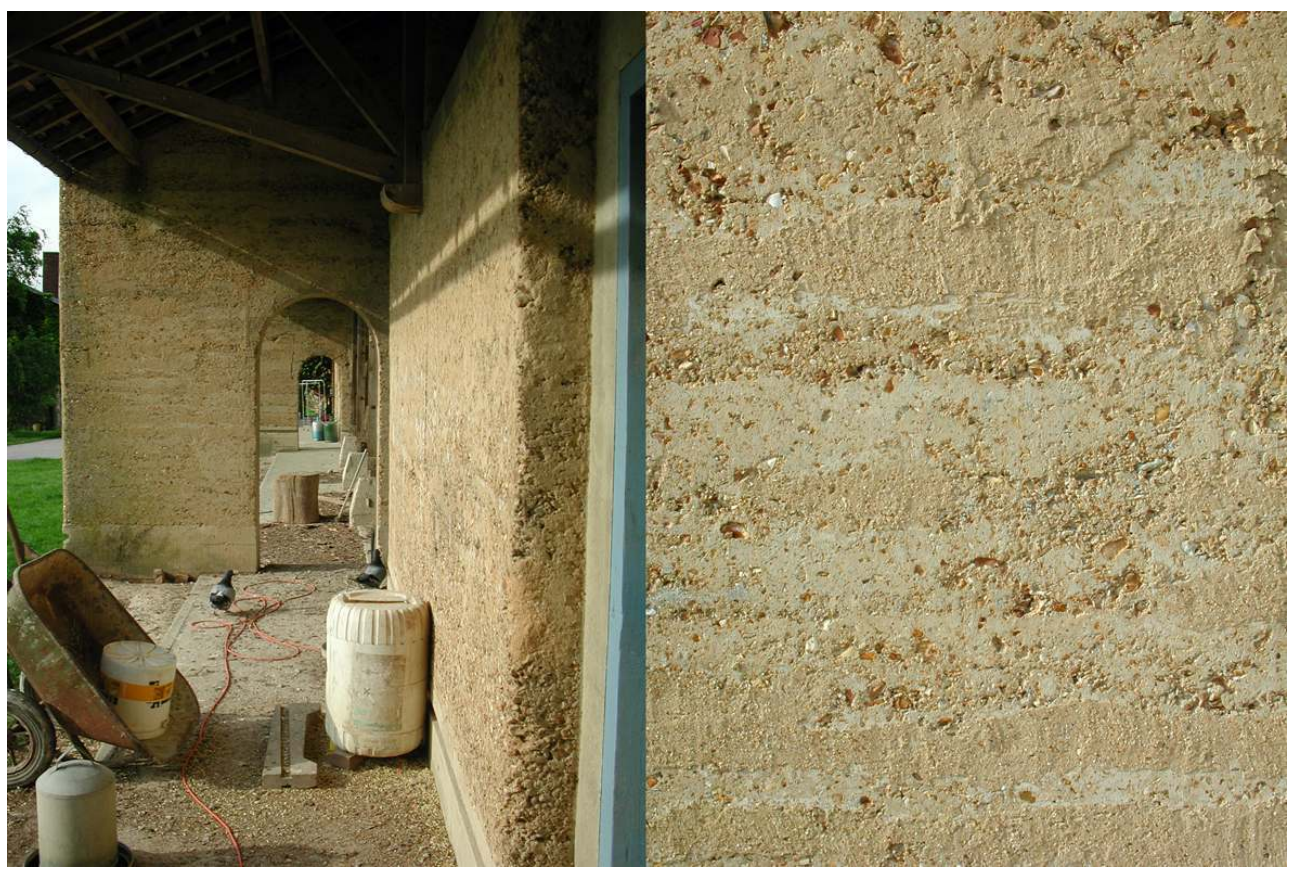

Le béton de terre des annexes de la ferme Quesnel. Vues contemporaines. 20 mai 2002.

Phot. Dousson, Xavier. (c) Xavier Dousson.

Pour répondre à la pénurie de matériaux, les maîtres d'œuvre imaginent également l'emploi de «parpaings de briquaillons» fabriqués à partir des résidus de briques provenant de la démolition des immeubles sinistrés. Les premiers essais se révélant satisfaisants, ils sont, en avril 1946, sur le point d'être fabriqués en grande série ${ }^{52}$. Ils seront ainsi utilisés pour de nombreuses constructions du village, tant en remplissage qu'en structure. Comme pour le béton de terre, ils présentent un aspect grenu, mais dans une teinte où se mêlent le rouge de la brique, le gris du ciment et tous les beiges et bruns des différents agrégats, créant une sorte de rose-gris typique. Par ailleurs la variété des éléments réemployés a empêché toute homogénéité de teinte entre les divers parpaings produits, créant de fait, comme naturellement, une vibration colorée - elle aussi typique - sur les parois. C'est cette teinte et cette vibration, plus que celles du béton de terre finalement peu employé, qui marqueront - avec bien évidemment d'autres dispositifs techniques et solutions plastiques - la reconstruction du Bosquel (fig. $\mathbf{n}^{\circ} \mathbf{2 1}$ ). 


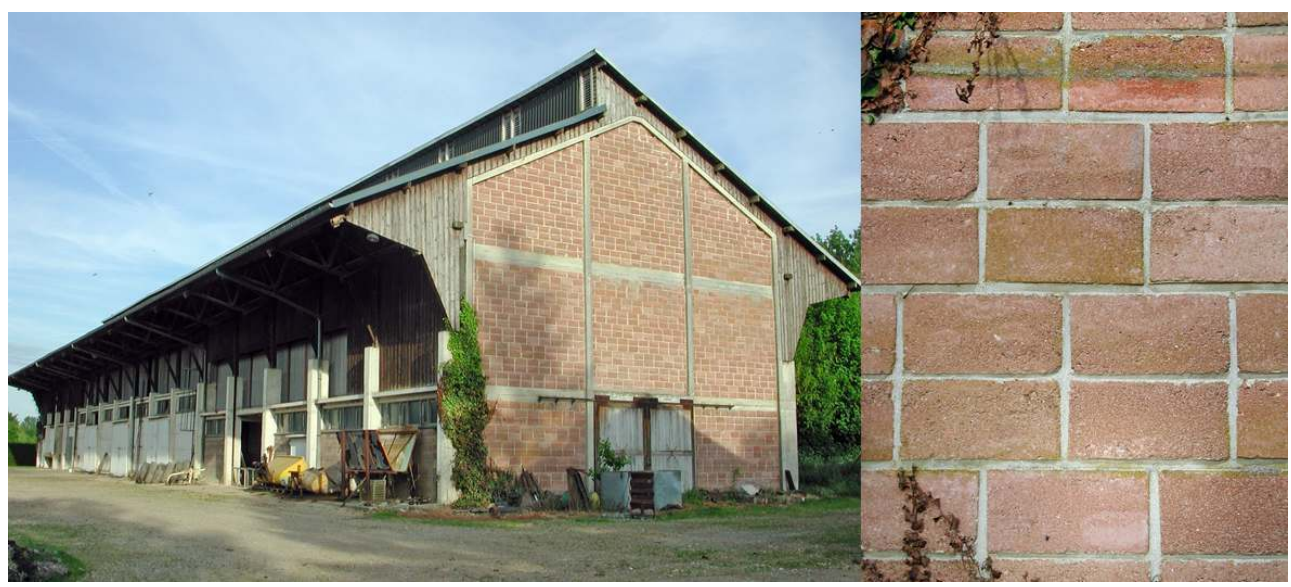

Les parpaings de briquaillons du bâtiment d'exploitation de la ferme Ropiquet. Vue d'ensemble et détail du mur pignon. 20 mai 2002.

Phot. Dousson, Xavier. (C) Xavier Dousson.

Au-delà de ces techniques constructives, ce qui marque profondément ce chantier est le travail très poussé des architectes en faveur d'une préfabrication légère et standardisée, produisant des éléments manutentionnables, les "constituants types $»^{53}$. Ces divers éléments (encadrements de portes et de fenêtres en béton armé, menuiseries, blocs de remplissage, dispositifs techniques, etc.) sont conçus pour être facile à fabriquer sur le chantier et transportables manuellement, sans l'aide de machines particulières (fig. $\mathbf{n}^{\circ} \mathbf{2 2}$ ) . En ce sens, ils permettent à la reconstruction de démarrer sans attendre un outillage technique complexe et coûteux, comme le nécessitera bientôt la préfabrication lourde. Ils sont surtout combinables les uns avec les autres, comme autant de composants articulés et réglés entre eux, à assembler à l'intérieur d'une trame constructive (gros œuvre, ossature), elle-même réglée et liée à eux. Cette manière de penser la construction, qui unit tous les éléments constructifs (ossature, remplissages, percements) en un seul système, permet aux architectes d'assurer à la fois une forte unité au projet de reconstruction du Bosquel tout en lui assurant une grande souplesse d'utilisation, autorisant toutes sortes d'adaptations et de variations. Comme l'écrit Jean Bossu, ce système de « constituants types » combine « l'unité des éléments employés et la diversité dans leur utilisation ${ }^{54} »$. Il va marquer toute une génération d'architectes, en particulier des personnalités comme Jacques Leperre, Louis Ouhayoun ou encore Paul Chemetov. 


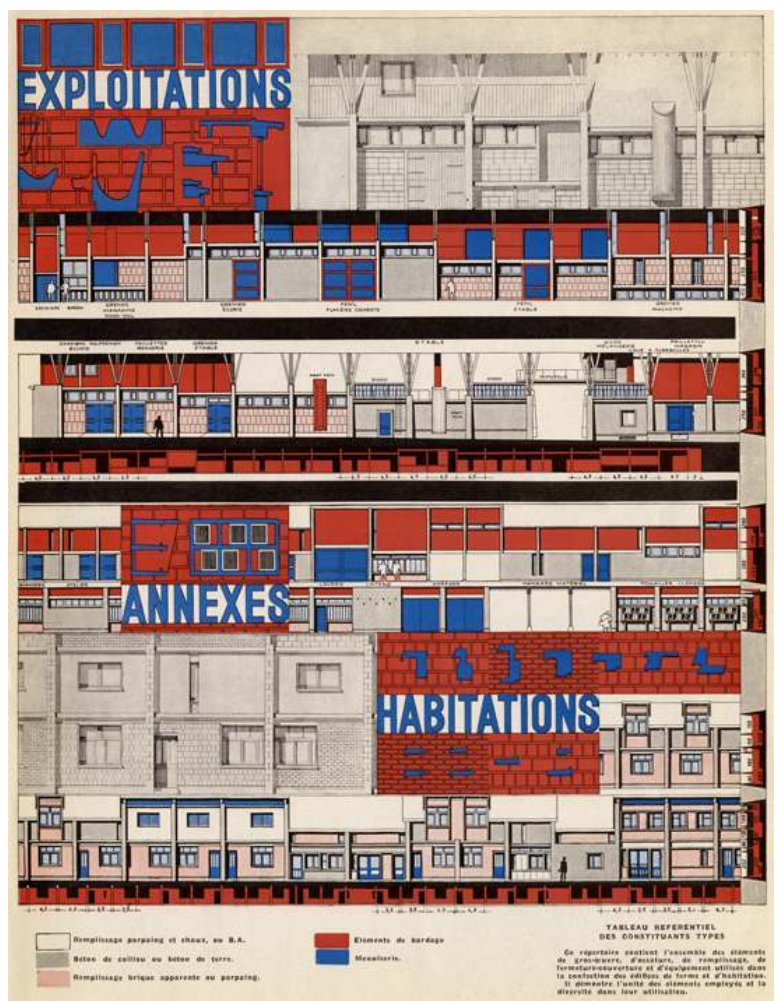

Les « constituants types » de la reconstruction du village témoin du Bosquel. Planche de présentation. BOSSU, Jean. « Le Bosquel, village de la Somme ». L'Architecture d'aujourd'hui, mars 1949, n²2, p. 51.

Repro. Dousson, Xavier. (c) Xavier Dousson.

\section{Une réussite paradoxale?}

En 1949, en pleine reconstruction, Jean Bossu peut donc conclure de l'ensemble des expériences menées au Bosquel qu'elles: «Démontrent clairement que les bâtiments ruraux doivent avoir pour caractéristique de permettre toutes les mutations nécessitées par la politique économique d'une exploitation ${ }^{55}$. » Pourtant, la complexité des bâtiments, en particulier de celui du bâtiment d'exploitation de la ferme Quesnel, dessinés jusqu'aux mangeoires des bêtes réalisées en béton armé, interdira ces transformations envisagées par les architectes. En fait, ces bâtiments, « conçus pour une période qui n'a pas eu lieu ${ }^{56}$ ", se révéleront obsolètes assez rapidement. Cette " obsolescence programmée ", ne doit pas nous empêcher de conclure, comme François Calame, Marie-Christine Zelem et Vintilà Mihailescu ${ }^{57}$, qu' : $^{\prime}$

« en réalité, la population du Bosquel a bénéficié, en son temps, des techniques de construction les plus nouvelles, d'une restructuration rationnelle de son espace de travail et de son habitat, d'une refonte complète de son parcellaire, dont on se félicite aujourd'hui. Elle a, par contre, mal vécu la rupture historique qui a frappé le monde agricole à partir de 1950. Cette véritable révolution dans l'économie et les techniques était-elle prévisible au moment où a débuté ce projet? C'est en fait plutôt un défaut d'assistance de la part de l'État face à cette évolution qu'ont déploré ces ruraux accoutumés à un traitement de faveur de la part de l'administration.» 
41 La réussite indéniable de cette reconstruction tient principalement dans sa capacité à avoir produit, en quelques années seulement, un cadre matériel unitaire et singulier à la vie rurale du village. Ce cadre a permis de dépasser le traumatisme infligé par les destructions de la guerre et le bouleversement induit par le nouveau remembrement tout en améliorant spectaculairement les conditions de travail, de vie et la production agricole. Surtout, il a donné aux habitants un village unique, à la fois éloigné de toute reconstruction formelle à l'identique (de tout décor), de presque toute référence à l'histoire antérieure du lieu (et ses stratifications induites par le parcellaire et la succession dans le temps des interventions humaines), sans pour autant être une abstraction contemporaine, nous dirions aujourd'hui " une architecture générique ». Ce village si identifiable, apparaît, pour reprendre les termes de l'architecte Hubert Lempereur ${ }^{58}$, comme une sorte de "condensation du temps ", un exemple fascinant à l'« étrange pouvoir d'attraction », d'autant plus affirmé qu'il n'a pas fait depuis l'objet de modifications importantes (pas d'extension, ni d'arrivée de zone d'activité ou commerciale).

42 Ni régionaliste, ni réponse universelle, la reconstruction de ce village pourrait être rapprochée de l'idée de « régionalisme critique » définie par Kenneth Frampton ${ }^{59}$. Cette spécificité n'a pas échappé à la critique architecturale et aux historiens : la reconstruction du Bosquel apparaît comme un moment important de la Reconstruction en France ${ }^{60}$.

\section{NOTES}

1. - Pour Marie-Christine Zelem, Dufournet «avait déjà réuni un nombre considérable de documents sur les villages picards en vue d'une thèse de l'Institut d'urbanisme ». ZELEM, MarieChristine. Reconstruction ou modernisation? Un village après la tempête : le Bosquel en Picardie. Mission du patrimoine ethnologique, ministère de la Culture et de la Communication, octobre 1991, p. 30. Cette recherche est disponible en ligne : <lien vers http://www.culturecommunication.gouv.fr/ index.php/content/download/44649/354830/file/Ethno_Zelem_1991_311.pdf> Plusieurs publications attestent de cette connaissance de l'urbaniste, en particulier : DUFOURNET, Paul. «Comment se sont construits les villages picards et ce qu'il en advient ». Techniques \& Architecture , $3^{\mathrm{e}}$ année, $\mathrm{n}^{\text {os }} 11-12$, novembre-décembre 1943, p.323-361 (numéro spécial «Techniques locales»). Ce texte a été republié, dans DUFOURNET, Paul, en collaboration avec CALAME, François. Itinéraire en architecture rurale, Amiénois Artois Boulonnais Ponthieu (1933-1948). Lillers, Saint-Valery-sur-Somme : Sépia/Chemins de traverses éditeur, DRAC Picardie, 1991, p. 105-122.

2. - Pour reprendre les termes du Progrès de la Somme, 4 novembre 1942.

3. - L'histoire de ce projet est relatée en détail par Paul Dufournet en 1991. «Reconstruction du Bosquel ». Dans Itinéraire en architecture rurale, op. cit., p. 142-148.

4. - DUfOURNET, Paul. Le Bosquel, Somme, rapport justificatif. 10 septembre 1941. Commissariat technique à la reconstruction immobilière, document dactylographié de 39 pages.

5. - «Tel qu'il est actuellement, ce village (les bourgs et les petites villes mises à part, naturellement) est le plus détruit du département de la Somme. Peut-être même est-il parmi les villages français, celui qui a le plus souffert, relativement à son importance et au nombre de ses 
maisons. » DUFOURNET, Paul. Le Bosquel, Somme, rapport justificatif, op. cit., p.13-14. Les publications de l'époque font état d'une destruction de $90 \%$ à $95 \%$ du village.

6. - Il écrit : «Le village se compose non seulement de maisons, mais de chemins, de jardins, de pâtures, de vergers, d'arbres ; tout ceci est intact, et représente une grande valeur. C'est une véritable construction, une modélation séculaire du sol, qui isole l'homme de son milieu et le protège contre le froid, le vent, l'humidité, la solitude. Il faudrait bien longtemps pour en retrouver l'équivalent. » DUFOURNET, Paul. Ibid., p. 19.

7. - « Je propose, en effet, que cette distribution soit souterraine, car rien n'abîme davantage nos villages de France que ces poteaux, potences et fils qui suivent les rues, et tombent toujours, comme par hasard, aux points les plus intéressants. Cela coûtera trop cher dit-on. Mais pourquoi ne pas en faire ici l'expérience et en tirer des enseignements qui permettront peut-être ensuite de le réaliser plus économiquement ? » DUFOURNET, Paul. Ibid, p. 15.

8. - CALAME, François, MIHAILESCU, Vintilà et ZELEM, Marie-Christine. « Picardie/Valachie : des villages après la tempête ». Terrain, octobre 1991, n 17, p. 154.

9. - Un premier remembrement avait été effectué moins de 10 ans auparavant (en 1934) sur le finage. Sa portée, reconnue ensuite par les habitants, avait été limitée compte tenu du fait des nombreuses controverses qu'il avait suscité. Dufournet écrit : « La première étape doit consister dans un remembrement ». Le Bosquel, Somme, rapport justificatif, op. cit., p. 27.

10. - Comme dans cette citation: «Le plan de reconstruction et d'urbanisme fut déclaré d'utilité publique au début de 1943. Le service départemental du Génie rural chargea alors M. Gay, géomètre expert, de procéder à ce nouveau remembrement, finage et agglomération confondus. On alla même plus loin, ne considérant que les exploitants, non les propriétaires, en regroupant dans le domaine des premiers les terres qu'ils tenaient en location. Le but était de parvenir à une organisation procurant le maximum de résultats aux points de vue agricole et humain.» DUFOURNET, Paul. Itinéraire en architecture rurale, op. cit., p. 142.

11. - Extrait du procès-verbal de la conférence du 20 juillet 1942 à Amiens entre les services civils. Archives de Fontainebleau, versement du ministère de l'Équipement sous le n ${ }^{\circ}$ 820159/AFU 10788. Informations dans LABBE, Fabienne. L'Architecte Louis Miquel, 1913-1987. Mémoire de maîtrise d'art contemporain, sous la direction de Bruno Foucart et Françoise Hamon. Université de Paris IV-Sorbonne, 1990-1992, p. 76.

12. - Paul Dufournet écrit : "La seconde partie essentielle de l'aménagement consiste en la création d'une place et du regroupement dans ce lieu des services publics du village. [...] La nécessité de créer une place, centre de la vie communale, est bien certaine. " Le Bosquel, Somme, rapport justificatif, op. cit., p. 23.

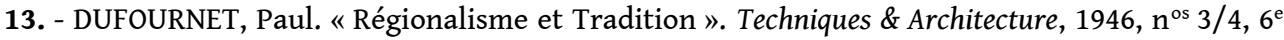
année, p. 152.

14. - Paul Dufournet précise : «Le village s'est construit à une époque lointaine, mais sur un tracé parcellaire bien plus ancien encore. Chacun, sur son étroit domaine, s'est organisé comme il a pu. Ne pouvant évoluer dans sa cour avec des voitures, il a placé la grange sur la rue. Naturellement, son habitation a été reléguée au fond et les écuries, étables et autres dépendances, ont pris, soit l'un, soit les deux autres côtés de la cour suivant les besoins et l'espace disponible. Le terrain était si étroit que toutes les granges se sont juxtaposées sur une ligne continue. [...] Le résultat a été ces rues longues, bordées de murs de torchis, uniquement percés par les portes de grange. Toute vie s'est cachée dans la cour et la famille a été isolée de son milieu. »Le Bosquel, Somme, rapport justificatif, op. cit., p. 28 . Voir, pour le photogramme du documentaire, figure $n^{\circ} 3$, le site : http://www.dailymotion.com/user/fonds-ancien/1\#video=xx5tx8

15. - Ce remembrement permet de passer de 529 à 69 parcelles. LABBE, Fabienne. L'Architecte Louis Miquel, op. cit., p. 76. Par exemple, la ferme Ropiquet, la plus grande exploitation, qui avait près de 60 parcelles dispersées sur tout le territoire communal avant le premier remembrement de 1934, n'en a plus qu'une après 1945. Informations dans BOSSU, Jean. «Le Bosquel: 
préliminaires de la reconstruction et premières réalisations ». Techniques \& Architecture, 1946, $\mathrm{n}^{\text {os }}$ 3/4, $6^{\mathrm{e}}$ année, p. 132.

16. - AUZELLE, Robert, DUFOURNET, Paul. « Le Béton de terre stabilisé ». Techniques \& Architecture

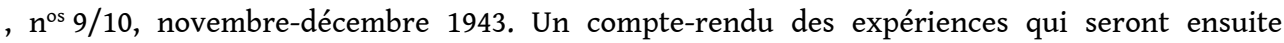
réalisées au Bosquel sera publié ultérieurement: DUFOURNET, Paul. "Une expérience de construction en béton de terre stabilisé ». Cahiers du Centre scientifique et technique du bâtiment, 2 ème trimestre $1950, \mathrm{n}^{\circ} 81, \mathrm{p}$. VII-XXIII. Ce texte est précédé d'une introduction « Le béton de terre ", non signée, qui replace l'expérience du Bosquel dans un contexte plus large prolongé par un texte non signé également de " projet de Mémento sur la mise en œuvre du béton de terre pour la construction des «bancos»". Le texte de Dufournet est par ailleurs reproduit dans Itinéraire en architecture rurale, op. cit., p. 149-164.

17. - Paul Dufournet écrit: «Le remembrement de base étant prêt dès avant la Libération, M. Raoul Dautry, alors ministre de la Reconstruction et de l'Urbanisme, décida, en février 1945, la reconstruction immédiate de ce village, à titre expérimental. » DUFOURNET, Paul. "Une expérience de construction en béton de terre stabilisé ».op. cit., p. VII.

18. - Lettre au chef du Service des matériaux, des transports et des constructions provisoires du MRU, le 22 février 1945. Archives départementales de la Somme, cote $15 \mathrm{~J} 11$.

19. - Ce deuxième point est l'argument principal retenu par Paul Dufournet pour justifier du choix de ces architectes. En effet, à notre connaissance, au moins trois d'entre eux ont participé pendant la guerre au chantier EAR 1425 (comme Enquête d'architecture rurale) du musée des Arts et Traditions populaires, une mission scientifique sous la direction de Georges-Henri Rivière, Pierre-Louis Duchartre, Urbain Cassan, Pierre Drobecq et Guy Pison: Jean Bossu en Vendée, Deux-Sèvres et Vienne, Louis Miquel dans les Alpes-Maritimes et les Basses-Alpes et Raymond Sénevat en Basse-Normandie. Raymond Sénevat en a même tiré une thèse. SENEVAT, Raymond. Urbanisme rural en bocage normand, canton de Brecey. Institut d'urbanisme de Paris, 1944, Bahrmann, président du jury. Par ailleurs, le numéro de Techniques \& Architecture déjà mentionné de novembre-décembre 1943, $\mathrm{n}^{\text {os }} 11 / 12$, spécial «techniques locales» sera essentiellement consacré à ce chantier 1425, avec des contributions substantielles de Jean Bossu (p. 280-287) et Raymond Sénevat (p. 304-311). Comme Dufournet contribue lui aussi à ce numéro, accompagnant son article de plans du Bosquel, nous supposons que les maîtres d'œuvres de la reconstruction du Bosquel se connaissent dès ce moment, voire même qu'ils travaillent déjà ensemble sur ce projet. 20. - DUFOURNET, Paul. «Reconstruction du Bosquel ». Itinéraire en architecture rurale, op. cit., p. 145.

21. - La position de Dufournet est probablement plus ambiguë. Dans son rapport de septembre 1941, celui-ci écrit : «L'aspect lui-même [de l'architecture] restera traditionnel. En effet, quoi de plus simple et de mieux adapté au caractère rural que cette architecture picarde, aux volumes simples et bien percés, et aux belles toitures. » Plus loin, il préconise d'employer la brique, de maintenir les couvertures traditionnelles (en tuile du Nord) et de faire « revivre la tradition locale des girouettes en fer découpé, représentant des scènes de la vie champêtre ", etc. (p. 30-31) Dans le même document pourtant, énumérant les éléments qui composent les habitations et les exploitations, il préconise de recourir à une forme d'industrialisation du bâtiment. Il écrit : «Ces éléments pourraient être parfaitement définis et produits de façon systématique.» (p. 37) Son ambition principale, la recherche d'unité, apparaît plus explicitement à la fin du document. Elle sera respectée par les architectes d'opération et rendue possible, entre autres, par le travail d'industrialisation et de répétition typologique de cette reconstruction. Paul Dufournet écrit : « Esthétique - La caractéristique principale de l'Architecture rurale dans une région est sa grande unité. La maison type se répète en un nombre infini d'exemplaires. Partout le même plan, la même constitution de l'ossature (murs et charpente) et les mêmes matériaux. La variation ne vient que du détail et de l'adaptation aux conditions particulières du terrain et des besoins. » (p. 38-39). Le Bosquel, Somme, rapport justificatif, op. cit.. En réalité, ce rapport de circonstance, écrit 
pour séduire les autorités de Vichy, ne reflète peut-être pas intégralement les véritables intentions architecturales de l'urbaniste.

22. - LE CORBUSIER. L'Urbanisme des trois établissements humains. Paris : coll. « les Cahiers forces vives » dirigées par Jean Petit, Les éditions de Minuit, 1959, p. 94. Voir également LE CORBUSIER et JEANNERET, Pierre. «Réorganisation agraire, 1934 ». L'Euvre complète, volume 2, 1929-1934. Zurich: publiée par Willy Boesiger et Oscar Stonorov, Erlenbach, éditions Girsberger, 1934, p. 186-191 ; LE CORBUSIER et JEANNERET, Pierre. «Réorganisation agraire, Le village coopératif, 1934-1938 ». L'Euvre complète, volume 3, 1934-1938. Zurich: publiée par Max Bill, éditions Girsberger, 1938, p. 104-115 ; LE CORBUSIER. « $7^{\mathrm{e}}$ Partie : réorganisation rurale ». La Ville radieuse, soleil, espace, verdure. Paris : éditions Vincent, Fréal \& Cie, 1933, p. 319-337.

23. - Citation de Le Corbusier extraite du numéro spécial de Techniques \& Architecture, entièrement consacré au thème de "la ferme ", novembre-décembre 1942, $\mathrm{n}^{\text {os }} 11 / 12,2^{\mathrm{e}}$ année, p. 397. Ce numéro est par ailleurs entièrement préparé par André Wogenscky, collaborateur de Le Corbusier depuis 1936.

24. - Le Corbusier écrit, sur la page de garde de la Ville radieuse: "Les plans ne sont pas de la politique. Les plans sont le monument rationnel et lyrique dressé au centre des contingences. Les contingences sont le milieu : régions, races, cultures, topographies, climats. Ce sont, d'autre part, les ressources apportées par les techniques modernes. Celles-ci sont universelles. Les contingences ne doivent être évaluées qu'en fonction de l'entité "homme", que par rapport à l'homme, que par rapport à nous, à nous autres : une biologie, une psychologie. » LE CORBUSIER. La Ville radieuse, soleil, espace, verdure, op. cit.

25. - Jean Bossu est le principal promoteur, avec Dufournet, de l'opération du Bosquel dans la presse spécialisée de l'époque. L'ensemble des publications qu'il rédige l'est dans une veine corbuséenne dont les citations ci-après rendent compte : « La ferme doit pouvoir se surveiller et se commander comme un atelier industriel ou un vaisseau. Elle doit posséder un poste de commande qui permette un contrôle rigoureux et rationnel.» BOSSU, Jean. «Le Bosquel: préliminaires de la reconstruction et premières réalisations ». Techniques \& Architecture, 1946, $\mathrm{n}^{\text {os }}$ 3/4, p. 137 ; « La ferme de l'époque machiniste doit être une usine. L'architecte doit trouver une organisation rationnelle du plan.» BOSSU, Jean. «Le Bosquel, prototype du village français ». Bâtir la France, organe de l'Union des architectes français, 5 décembre 1946.

26. - Le Bosquel, résultats d'enquêtes effectuées auprès des fermiers, note dactylographiée de 8 pages, non datée, réalisée par l'UTA (Union du technicien et de l'architecte), groupe fondé par les collaborateurs des architectes d'opération (Fabien Vienne, Christian Trudon, Pierre Sagui, etc.). Archives privées de Fabien Vienne.

27. - Au moment de la parution des premières réalisations de l'opération dans les pages de l'Architecture d'aujourd'hui, Jean Bossu peut tout de même conclure légitimement, et avec une pointe de fierté, que: "L'ensemble des dispositions adoptées est la conclusion d'une série d'enquêtes rigoureuses faites auprès des Services agricoles, du Génie rural et des cultivateurs. » BOSSU, Jean. «Le Bosquel, village de la Somme ». L'Architecture d'aujourd'hui, mars 1949, ${ }^{\circ} 22$, p. 52.

28. - Comme l'indique l'en-tête des courriers de l'époque, SIAF-CAPA - Archives d'architecture du $\mathrm{xx}^{\mathrm{e}}$ siècle, Fonds Jean Bossu, 192 ifa 36/2.

29. - Voir le plan de situation des fermes - implantation des constructions de la commune du Bosquel, daté du 5 novembre 1945. Ce plan est signé Dufournet, Dupré, Grandjean, Bossu, Sénevat et Miquel. Voir également le plan de situation des bâtiments de la ferme Quesnel, daté du 9 juillet 1945 et signé des mêmes auteurs. SIAF-CAPA - Archives d'architecture du XX ${ }^{\mathrm{e}}$ siècle, Fonds Jean Bossu, 192 ifa 36/1 et 192 ifa 38/1.

30. - Lettre du 20 février 1946 de Paul Dufournet à Bossu, Sénevat, Miquel et Dupré. Archives départementales de la Somme, côte 15J 12. 
31. - Lettre de démission adressée à Paul Dufournet. Archives départementales de la Somme, côte 15J 12. On n'y trouve pas d'explication de cette décision.

32. - Extrait de la pétition de quelques sinistrés du Bosquel au ministère des Finances et de l'Économie nationale au début de 1946. Archives de Fontainebleau, versement du ministère de l'Équipement sous le $\mathrm{n}^{\circ}$ 820159/AFU 10788. Information dans LABBE, Fabienne. L'Architecte Louis Miquel, op. cit., p. 77.

33. - « Celui-ci comprend, sous un grand parapluie de charpente, au rez-de-chaussée le logement des animaux, et au-dessus, celui des récoltes. C'est ici l'usine agricole proprement dite. Le foin, les paillettes, les grains tombent directement là où on en a besoin. » BOSSU, Jean. « Le Bosquel, village de la Somme ", l'Architecture d'aujourd'hui, op. cit., p. 50.

34. - Pour reprendre les termes des architectes. «Le Bosquel - chantier $n^{\circ} 1$ ». Techniques \& Architecture, 1946, $\mathrm{n}^{\text {os }} 7 / 8,6^{\mathrm{e}}$ année, numéro spécial Reconstruction, p. 379.

35. - « Le Bosquel - chantier $n^{\circ} 1 »$. op. cit., p. 379.

36. - Entretiens avec Fabien et Jacqueline Vienne le mardi 24 avril 1997 et avec Pierre Sagui le mardi 13 mai 1997, anciens collaborateurs de Jean Bossu.

37. - Archives départementales de la Somme, côte $15 \mathrm{~J}$.

38. - La maquette de la ferme Quesnel renseigne sur l'orientation prévue originellement. Celle-ci était l'inverse de celle finalement retenue : vers le village et vers le soleil levant. En reprenant l'étude de la maison, Jean Bossu a retourné la maison vers la cour, la plus belle vue et le soleil couchant, privilégiant son rapport avec les autres constructions de la ferme Quesnel.

39. - L'administration ne s'est d'ailleurs pas trompée sur cet état de fait, proposant le classement à l'ISMH de l'ensemble de la ferme en 1989 (rapport de Vincent Brunelle, architecte en chef des monuments historiques, Arras, le 19 septembre 1989). Ce sont les actuels propriétaires des lieux, pourtant très sensibilisés à cette conservation, qui ont refusé son classement devant les contraintes qu'il génère et le peu d'aides financières qu'il apporte, préférant entreprendre par eux-mêmes la réfection progressive des bâtiments en vue d'une reconversion. Aujourd'hui, le bâtiment d'exploitation de la ferme Quesnel sert partiellement de salle de réunion (mariages, banquets, fêtes, etc.) et peut-être loué à la demande. Par ailleurs, les annexes ont été transformées en chambres d'hôtes.

40. - Une photo d'une maquette d'une charpente tridimensionnelle de l'ingénieur introduit l'article « Le Bosquel : préliminaires de la reconstruction et premières réalisations ». Techniques \& Architecture, op. cit., p. 131. Par ailleurs, ce numéro spécial «Aménagement rural » présente surtout un article intitulé «Hangars agricoles», p. 126-127, qui détaille sur une même page le hangar de la ferme Quesnel en construction et le système Aplex de Le Ricolais.

41. - DUFOURNET, Paul. «Reconstruction du Bosquel ». Itinéraire en architecture rurale, op. cit., p. 145.

42. - Épure de statique graphique.

43. - Le nom de Gauthier, sur lequel nous n'avons pas trouvé d'autres renseignements, est cité dans Techniques \& Architecture, $\mathrm{n}^{\text {os }} 3 / 4$, op. cit., p. 127 et 133, ainsi que dans Techniques \& Architecture, $\mathrm{n}^{\text {os }} 7 / 8$, op. cit., p. 379.

44. - À l'exception de la ferme Derogy dont le bâtiment d'exploitation dévoile une charpente en béton armé.

45. - Et qu'il réalisera au Cameroun en 1948. Voir : l'exposition «L'Art de l'ingénieur », sous la direction d'Antoine Picon, présentée de juin à septembre 1997 au Centre Pompidou et qui montrait plusieurs maquettes et photographies du système Aplex réalisé au Cameroun par Le Ricolais, avant son départ aux États-Unis en 1951.

46. - Trois travées sud de la charpente du bâtiment d'exploitation de la ferme Quesnel ont été déposées dans le courant des années 1990. Non entretenues pendant près de 50 ans, ainsi que la toiture qui montrait de nombreux signes d'infiltration, elles menaçaient de s'effondrer. Il est remarquable qu'elles aient tenu sans aucun entretien pendant tout ce temps. Il est tout aussi 
remarquable de constater qu'elles n'ont pu être remplacées, trop complexes et trop chères à reconstruire, ce qui indique leur raffinement. Témoignage de l'actuel propriétaire et exploitant de la ferme Quesnel, Pascal Dacheux, petit-fils des maîtres d'ouvrage.

47. - Nous ne savons pas si, au-delà du système Aplex, Le Ricolais a participé à d'autres études pour ce projet et, en particulier, s'il a influencé directement la conception des charpentes finalement retenues.

48. - Il en faut environ cinq fois plus dans le cas d'un mur en béton.

49. - DUFOURNET, Paul. « Une expérience de construction en béton de terre stabilisé ». Cahiers du Centre scientifique et technique $d u$ bâtiment, op. cit. Ce long texte renseigne l'ensemble de l'expérience du Bosquel en matière de constructions en béton de terre stabilisée. Il peut être utilement consulté pour tout renseignement supplémentaire.

50. - Rien qu'en France, un laboratoire de recherche de l'École nationale supérieure d'architecture de Grenoble (ENSAG) est entièrement dédié à l'étude, la valorisation et l'expérimentation des techniques de construction à base de terre : Le CRA-Terre, fondé en 1979 est habilité depuis 1986 par la DAPA (direction de l'Architecture et du Patrimoine). Ce laboratoire a produit de nombreuses publications, recherches et expositions, dont plusieurs sont disponibles sur internet < lien vers http://craterre.org/ > Il a depuis longtemps détecté l'importance du projet de reconstruction du Bosquel en matière de construction en béton de terre. Voir, par exemple : DOAT, Patrice, GUILLAUD, Hubert, ROLLET, Pascal et HOUBEN, Hugo. Pour une étude raisonnée des architectures en pisé, étude du savoir-faire " pisé " français et étranger. Rapport final de recherche, Grenoble, 1985, 388 pages, p. 68-98.

51. -Voir, par exemple, le travail remarquable de Rick Joy. JOY, Rick. Desert Works. New York: Princeton Architectural Press, 2002. Voir également l'ensemble des édicules en béton de terre du récent parc de Gerland à Lyon de Michel Corajoud (2000).

52. - Lettre du 15 avril 1946 du délégué départemental du ministère de la Reconstruction et de l'Urbanisme au directeur du Cabinet de ce même ministère. Archives de Fontainebleau, versement du ministère de l'Équipement sous le $n^{\circ}$ 820159/AFU 10788. Informations dans LABBE, Fabienne. L'Architecte Louis Miquel, op. cit., p. 78.

53. - Voir, en particulier, le dessin qui exprime, comme une affiche séduisante, la richesse plastique des assemblages rendus possibles par ce système de « constituants types ». BOSSU, Jean. «Le Bosquel, village de la Somme ». L'Architecture d'aujourd'hui, op. cit., p. 51.

54. -Ibid., p. 51.

55. - Ibid., p. 52.

56. - Cette citation est une expression de l'actuel propriétaire et exploitant de la ferme Quesnel, Pascal Dacheux, petit-fils des maîtres d'ouvrage, agriculteur bio et élu écologiste en Picardie. Elle indique que cette période, celle d'une exploitation rationnelle de la terre et de l'élevage, mais avec une main-d'œuvre abondante et une force motrice essentiellement hippomobile, n'a eu lieu qu'à peine une quinzaine d'années. Les bâtiments du Bosquel, tellement adaptés aux modes de faire d'avant la mécanisation, au point d'être considérés comme des modèles, n'ont pas anticipé les bouleversements de l'agriculture durant les « Trente Glorieuses». Voir également sur ce sujet les témoignages des agriculteurs ou exploitants du Bosquel recueillis par Marie-Christine Zelem dans Reconstruction ou modernisation? Un village après la tempête, op. cit. : Comme celui-ci, p. 94 : «Au départ, ça a été dur. Mais tout ça, ça s'est fait peu à peu... et puis la génération de l'époque a disparu. Tout a tellement changé. Avant, c'étaient des attelages partout, aujourd'hui... c'est fini. Ils ont fait des écuries avec des râteliers impeccables. Ils n'ont jamais servi! Pour mettre les chevaux et tout ça, ça n'a jamais servi! Aujourd'hui, on ne peut même pas rentrer un tracteur dans ces bâtiments. Les plafonds ne sont même pas assez hauts pour rentrer du matériel. Ils sont pourtant immenses et coûtent une fortune en entretien. Le Bosquel a une guerre de retard! Les soi-disant "fermes de l'avenir" ont été dépassées avant d'avoir servi ! » Ou encore, p. 100 : «Il y avait des troupeaux de moutons avant. Ils allaient au parc. Il n'y en a plus maintenant ! La culture 
a changé aussi. Dans la plupart des fermes, ils ont fait des bâtiments qui n'ont pratiquement pas servi. On a eu nos écuries quand il n'y a plus eu de chevaux!»

57. - CALAME, François, MIHAILESCU, Vintilà et ZELEM, Marie-Christine. «Picardie/Valachie : des villages après la tempête ", op. cit., p. 158.

58. - Hubert Lempereur emploie ces termes dans un article éclairant à propos d'un village comparable, reconstruit également sous la direction de Paul Dufournet, mais avec d'autres architectes d'opération. LEMPEREUR, Hubert. «Bernes-sur-Oise, une reconstruction ». AMC, février 2007, nº 167, rubrique « Référence », p. 78-85.

59. - FRAMPTON, Kenneth. « Chapitre 6 : post-scriptum 1983. L'architecture contemporaine et le régionalisme critique ». L'Architecture moderne, une histoire critique. Paris : Philippe Sers éditeur, 1985, p. 276-296.

60. - Citons, en particulier : LUCAN, Jacques. Architecture en France (1940-2000), histoire et théories. Paris : coll. «Architextes" Le Moniteur, p. 30-32 ; VOLDMAN, Danièle. La Reconstruction des villes françaises de 1940 à 1954, histoire d'une politique. Paris, Montréal : éditions l'Harmattan, 1997, p. 298-299 ; LABBE, Fabienne. «La reconstruction du Bosquel et de Tergnier au lendemain de la Deuxième Guerre mondiale ». Dans Villes reconstruites, $d u$ dessin au destin. Actes du deuxième colloque international des villes reconstruites, présentation de Patrick Dieudonné, 2 volumes, Ville de Lorient, Institut de géoarchitecture, éditions l'Harmattan, 1994, p. 218-225 ; CIVIDINO, Hervé. Architectures agricoles, La modernisation des fermes 1945-1999. Préface de Daniel Le Couédic. Rennes : Coll. « Art \& Société », Presses universitaires de Rennes, 2012, p. 150-152.

\section{RÉSUMÉS}

Le 7 juin 1940, le village du Bosquel est presque entièrement détruit par l'avancée de l'armée allemande. La tabula rasa provoquée par la guerre va permettre à son urbaniste, Paul Dufournet, et ses architectes d'opération de redéfinir complètement la structuration du village, en particulier la disposition de ses fonctions principales, son inscription dans l'espace rural ainsi que la nature et la forme de ses maisons et de ses fermes. Sur ce dernier point, la ferme Quesnel, la première réalisée, modèle ensuite de l'ensemble de la reconstruction du Bosquel, en rupture totale avec les anciennes fermes picardes, apparaît comme la figure la plus singulière de cette reconstruction.

On $7^{\text {th }}$ June 1940, the village of Bosquel was almost entirely destroyed by the advancing German army. The tabula rasa caused by the war meant that their Town Planner, Paul Dufournet, and his project architects were able to completely redefine the structuring of the village, particularly the placement of its key functions, its inclusion in the rural area, as well as the character and form of its houses and farms. Regarding this last point, the Quesnel farm, the first one completed, became a model for the reconstruction of Bosquel as a whole making a complete break with the former farms of Picardy, and appears as the most singular figure in this reconstruction.

\section{INDEX}

Mots-clés : Paul Dufournet, Jean Bossu, Louis Miquel, Robert Le Ricolais, Le Corbusier, Reconstruction, béton de terre, charpente tridimensionnelle, village prototype, village témoin 


\section{AUTEUR}

\section{XAVIER DOUSSON}

Architecte. Chercheur au LACTH, École nationale supérieure d'architecture et de paysage de Lille. Maître-assistant associé à l'École nationale supérieure d'architecture Paris-Malaquais xdousson@club-internet.fr 